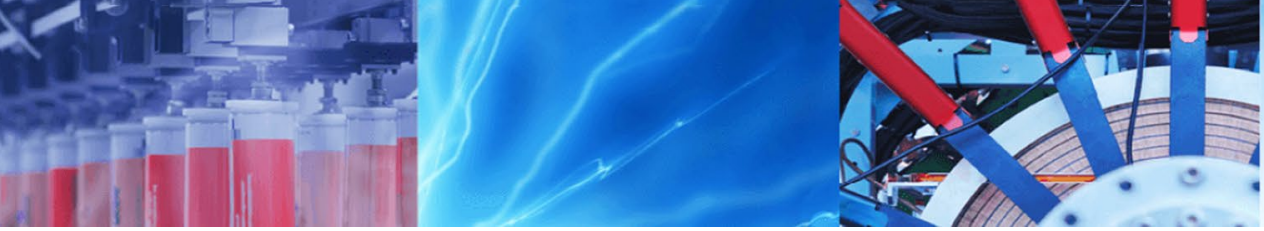

Research Article

\title{
Solving time-dependent heat conduction problems using metaheuristic algorithms extended with a novel local search strategy
}

\author{
Oguz Emrah Turgut ${ }^{1}$ (D)
}

Received: 6 July 2020 / Accepted: 22 December 2020 / Published online: 11 January 2021

(c) The Author(s) 2021 OPEN

\begin{abstract}
This study proposes a novel and dexterous local search scheme for improving the exploitation phase of a generic metaheuristic algorithm. The proposed local search considers a twofold probing mechanism, which takes advantage of a chaotic number generated by the hybrid chaotic map composed of Logistic map and Kent map to move around the so-far-obtained global best solutions to reach feasible candidate solutions. Also, an iterative local search scheme inspired by a variant of the differential evolution algorithm is incorporated into the proposed manipulation scheme to enhance intensification on the promising regions. The proposed scheme is included in the well-reputed metaheuristics of differential evolution, crow search, whale optimization, and sine-cosine algorithms to assess the resulting improvements made on the optimization accuracy. Forty optimization benchmark functions composed of unimodal and multimodal test problems have been solved by the local search improved and basic forms of these optimizers to identify the amelioration in terms of solution accuracy and robustness. Two different real-world constrained optimization problems have been solved by these algorithms to analyze the improvement in solution qualities maintained by the utilization of the proposed local search method. Furthermore, these mentioned optimization algorithms along with their improved forms have been applied to one-dimensional transient heat conduction problems to obtain accurate temperature distribution across the heat transfer medium. Optimization results reveal that utilizing local search enhanced metaheuristic algorithms can be considered a favorable alternative to conventional solution methods for solving transient heat conduction problems.
\end{abstract}

Keywords Local search · Heat conduction · Metaheuristics · Crow search

\section{Introduction}

Optimization is an iterative process that explores a search domain to obtain the optimal solution of the problem under the predefined specific conditions. In the context of mathematics, optimization aims to find the set of optimal decision variables $\left(x^{*}\right)$ which minimizes or maximizes the respective objective function value $f\left(x^{*}\right)$ depending on the nature of the optimization problem. Optimization algorithms are practical methods utilized in obtaining optimal values of the problems in many disciplines. Optimization methods can be divided into two major categories, which are namely deterministic and stochastic algorithms. Deterministic methods can provide and guarantee the optimal solution by using the analytical domain information of the corresponding problem. Deterministic methods ensure the global answer within the prescribed error tolerances after exhaustive and tedious time-consuming algorithm runs. However, they may collapse in generating optimal results of black-box and noisy optimization problems. Besides, most of the deterministic methods require good initial estimates and derivative information of the problem domain which complicates the acquisition of reliable candidate solutions. Stochastic optimizers are more flexible

Oguz Emrah Turgut, oeturgut@hotmail.com | 'Department of Industrial Engineering, Faculty of Engineering, Izmir Bakırcay University, Izmir, Turkey.

SN Applied Sciences (2021) 3:71 | https://doi.org/10.1007/s42452-020-04065-3 
and efficient than deterministic methods in dealing with black-box optimization problems. They are generally more suitable to tackle problems where domain knowledge of the objective function is not priorly known. Albeit, the major drawback of these types of algorithms is that global optimum is not guaranteed as they may get stuck in local optima in the search space due to the inherent inefficiencies of search equations [1].

This study deals with the useful application of metaheuristic algorithms, which is one of the most prominent members of stochastic optimization methods. Metaheuristic optimizers can also be subdivided into several branches based on their inspiration foundations such as evolutionary algorithms (EA), swarm intelligence algorithms, physics-based algorithms, etc. Genetic algorithms are the most popular and prevalent members of EAs, which imitates the major biological evolution concepts of living beings such as crossover, mutation, and selection [2]. Gravitational search algorithm [3] is inspired by the gravitational laws and principle laws of motion. This algorithm can be exemplified as an eminent member of physics-based algorithms. Particle swarm optimization [4] is the one of most famous swarm intelligence methods in the literature, which emulates the flocking behaviors of birds to reach the optimal solution to the problem.

Literature also comprises plenty of methodical procedures for improving the solution efficiency of the available metaheuristic algorithms. One frequently applied method is creating synergy between two or three algorithms by hybridization, which entails an improvement in the cumulative performance of the hybridized methods. The main idea behind this hybridization is eliminating the characteristic disadvantages of each optimizer through combining the salient features thereby, producing high-quality candidate solutions for upcoming iterations. Zhang et al. [5] proposed a novel hybridized algorithm called CS-DE for solving real-world constrained optimization problems. Cuckoo search (CS) has good abilities to perform global search thanks to unpredictable jumps on the search domain made by Levy flights. Differential evolution (DE) is known to be an efficient optimizer for finetuning the trial solution samples to obtain a globally optimum solution. Combining these optimizers conquers the characteristic algorithmic drawbacks of CS to some extent, which are premature convergence to local optimum points and lack of population diversity. Singh et al. [6] integrate the basic search equations of the Salp Swarm Algorithm with the perturbation scheme of the sine cosine algorithm for solving nonlinear high dimensional optimization problems. Hybridization occurs in such a way that each salp in the population updates its current position through the merits of sine and cosine functions. Numerical results on various types of benchmark functions divulge that incorporating these two algorithms entails an enhancement in exploration and exploitation capabilities of the hybrid method, which is two main generic features of any metaheuristic algorithm characterizing its solution accuracy and robustness. Another option to upgrade the solution accuracy is using the merits of deterministic randomness produced various types of chaotic maps, which can provide unpredictable random number sequences [7]. According to the past studies regarding the integration of chaos theory into metaheuristic algorithms [8-10], it is understood that using chaotic numbers instead of uniformly distributed Gaussian random numbers significantly boosts the exploration and exploitation phases of the base algorithm. There are many applications of chaotic numbers on the available metaheuristics such as gravitational search algorithm [11], artificial immune system [12] and dragonfly algorithm [13], etc.

Local search methods are another favorable alternative for optimization performance improvement. In different literature approaches, authors utilize and take advantage of varying types of local search procedures to polish the promising trial solutions obtained by the base algorithm. Moradi and Parsa [14] developed a genetic algorithm based evolutionary method for community detection. Trial solutions retained by the evolutionary algorithm is finetuned by a local search strategy to accelerate convergence and improve the diversity in the population. Qu et al. [15] integrate a novel local search scheme into the basic manipulation equations of particle swarm optimization to enhance the exploitation of the fertile regions on the search domain. Toksari [16] hybridized the ant colony optimization algorithm with Iterated local search method to acquire better solution outcomes for estimation of Turkey's domestic electricity consumption. Xia et al. [17] combined a three-stage novel search method composed of tabu detecting strategy, shrinking, and local learning strategies with Particle Swarm Optimization to overcome the algorithm-specific deficiencies of premature convergence and local minima entrapment. Yildiz and Topal [18] proposed a micro Differential Evolution Algorithm reinforced by the Directional local search mechanism for solving high dimensional optimization problems. Gao et al. [19] put forward a Discrete Harmony Search algorithm amended with an ensemble of local search strategies for solving urban traffic light scheduling problems. This research study aims to provide two different novelties to the literature. A novel local search algorithm is proposed consisting of two manipulative phases to increase to probing mechanism of the base metaheuristic algorithm. The proposed approach considers a two-phase local search procedure that merges some kind of iterated local search method ameliorated by a hybrid chaotic with a modified variant of differential evolution scheme perturbed by an iteratively 
adjusted algorithm parameter. Developed ensemble local search method is separately integrated into the basic search equations of differential evolution (DE) [20], Crow search (CROW) [21], sine-cosine algorithm (SINECOS) [22], and Whale optimization algorithm (WHALE) [23] to ameliorate their search efficiencies. A test suite of forty optimization benchmark functions has been applied to these algorithms along with their local search improved versions to observe the improvement in terms of solution accuracy and robustness. Another novelty is that this is the first application of metaheuristic algorithms over solving transient heat conduction problems. The time-dependent temperature distribution of different heat transfer mediums is obtained by the mentioned optimizers rather than tedious and exhaustive root finding methods. Corresponding numerical results are compared and the superiority of the solutions found by local search improved optimizers are verified. The remaining parts of this research study are organized as follows: Sect. 2 describes the essential search mechanism behind the proposed local search scheme. Section 3 provides the numerical experiments dealing with the exhaustive comparative study between local search enhanced and base metaheuristic methods founded on multidimensional optimization benchmark functions. Section 4 reports the optimum results obtained by the local search extended metaheuristics for two different real-world complex constrained engineering design problems. Section 5 explains the application of the proposed local search enhanced methods on solving transient heat conduction problems and Sect. 6 concludes this study with remarkable comments.

\section{The proposed ensemble local search scheme}

In this section, a novel local search strategy is introduced which is an ensemble of a modified iterated local search scheme and a variant of the DE algorithm assisted with an iterative perturbation parameter. The main idea behind developing this kind of local search mechanism is to accelerate the convergence tendencies of the base algorithm by probing around and exploiting the extracted promising and fertile search spaces. As mentioned in the Introduction section, the proposed local search in the current research study employs concurrently performing two different mutation mechanisms, those are also complementing each other regarding the required balance between diversification and intensification. The first phase of the local search benefits from an integrated chaotic map, which is a hybridization of Logistic and Kent maps. Literature studies dealing with this issue strongly emphasizes the importance of hybridized chaotic maps and their solution-based superiorities over single chaotic maps. Luo et al. [24] proposed a novel image encryption algorithm based on hybridized chaotic maps of Baker and Logistic map and obtained competitive results compared to the other state-of-art image encryption algorithms. Demir et al. [25] proposed a novel hybrid chaotic map by forming Gauss and Logistic maps and applied the sequence of chaotic numbers on the search equations of particle swam optimization to enhance the solution quality. Pan et al. [26] constructed a hybrid chaotic system built upon the nonlinear combination of Sine map, Tent map, and Logistic map and applied this hybrid map and deep neural network structure to develop an efficient image encryption algorithm. Demir et al. [27] also claimed the foregoing idea that hybrid chaotic maps provide better performance compared to traditional one-dimensional chaotic maps and their feasible application over metaheuristic algorithms is very rare. They proposed a hybrid mapping procedure formed by Logistic and Sine maps and developed an original chaotic optimization algorithm benefiting the merits of the ergodic number sequences generated by the hybrid map. The proposed algorithm is tested on various types of benchmark problems along with a constrained engineering design problem and its performance is verified against the well-reputed optimizers.

As in most of the literature approaches, the utmost aim in constructing a hybrid chaotic map is to achieve a higher performance in generating random numbers relying on their unpredictable semi-random behavior. Chaotic maps provide plentiful advantages over metaheuristic algorithms in terms of performance improvement. Chaotic numbers do not repeat themselves within the prescribed search range which increases the possibility of exploring the unvisited regions on the solution domain, thereby persistently maintaining local minima avoidance. In this study, a hybrid Logistic-Kent chaotic map is proposed for updating the current position of population members in a metaheuristic algorithm. Trial-and-error based procedure is followed during the hybridization process. Various kinds of chaotic maps available in the literature are tested and applied to this hybridization scheme however created synergy between these two chaotic maps provides the most satisfactory solution outcomes. Below given set of nonlinear mathematical equations describe the essentials of each chaotic map used in the hybridization process. Chaotic number production in Kent [28] chaotic map can be expressed by the following equation.

$\operatorname{Kent}\left(\theta, x_{t}\right) \rightarrow x_{t+1}= \begin{cases}x_{t} / \theta & \text { if } 0<x_{t} \leq \theta \\ \frac{1-x_{t}}{1-\theta} & \text { elseif } \theta<x_{t}<1\end{cases}$

where $\theta$ is a random number very close to 0.5 . Chaotic number sequences through Logistic map are generated by the following equation 
$\operatorname{Logistic}\left(\beta, y_{t}\right) \rightarrow y_{t+1}=\beta y_{t}\left(1-y_{t}\right)$

where $\beta$ is considered to be 4.0 in Eq. (2). The algorithmic procedure of the hybridized method is provided in the following structural form

\begin{tabular}{|l|}
\hline Algorithm $1-$ The proposed hybrid chaotic map \\
\hline function $\left[z_{t+1}\right]=$ Logistic_Kent $\left(z_{t}\right)$ \\
$\quad z_{t+1}=\bmod \left(\operatorname{Kent}\left(\theta, \mathrm{z}_{t}\right)+\operatorname{Logistic}\left(\beta, \mathrm{z}_{t}\right), 1.0\right)$ \\
end
\end{tabular}

The produced chaotic number $z_{t}$ will be used for updating the current position of the particle in the proposed local search scheme which can be expressed in Algorithm 2

\begin{tabular}{|c|}
\hline Algorithm $2-$ Chaotic position update scheme \\
\hline for $i=1$ to $N$ \\
for $j=1$ to $D$ \\
end $X_{\text {new }}^{i, j}=X_{\text {best }}^{j} \times z^{i, j}$ \\
end \\
\hline
\end{tabular}

In Algorithm 2, $N$ is the population size and $D$ is the dimensionality of the problem to be optimized. $X_{n e w}^{i, j}$ represents the updated position of the ith population member at the $j$ th dimension; $X_{\text {best }}^{j}$ is the so-far-obtained best solution at the $j$ th dimension, and $z$ is the chaotic number

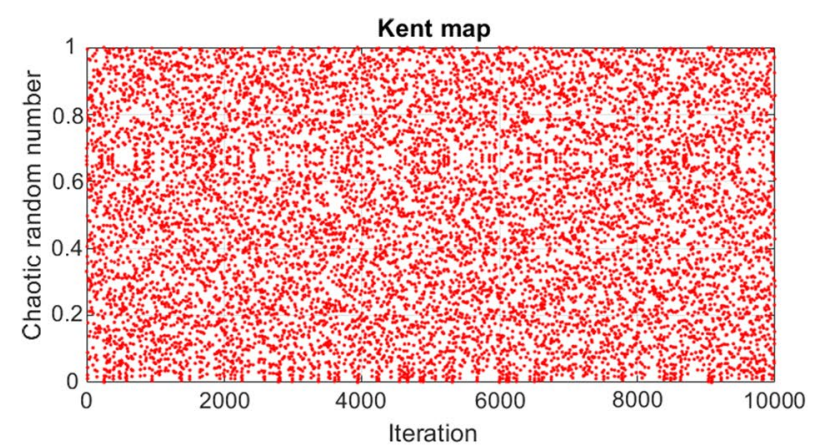

generated by the hybrid mapping. Relying on the unpredictable and ergodic behavior of the random numbers generated by the proposed hybrid scheme, search agents can effectively probe around the promising neighboring solutions and eliminate the pitfalls of local optimum points. Figure 1 visualizes the distribution of chaotic random numbers generated by the Logistic map, Kent map, and the proposed hybrid Kent-Logistic map. Figure 2 depicts the structural representation of the hybrid mapping procedure.

The second phase of the local search method is built upon a modified version of the DE variant called DE/best/1 whose respective perturbation equation is given below

$V_{i}=X_{\text {best }}+F\left(X_{r 1}-X_{r 2}\right)$

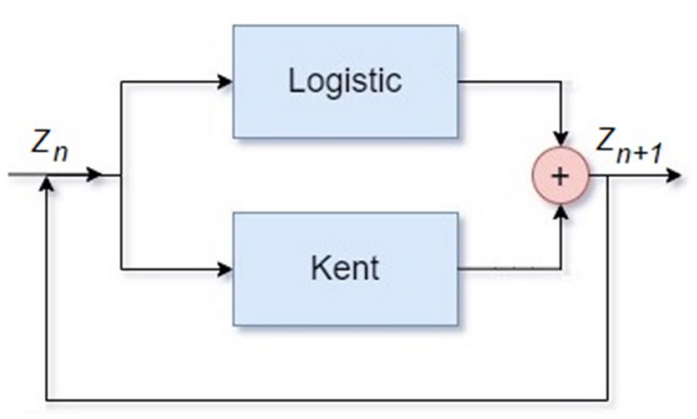

Fig. 2 Physical structure of the hybrid chaotic map
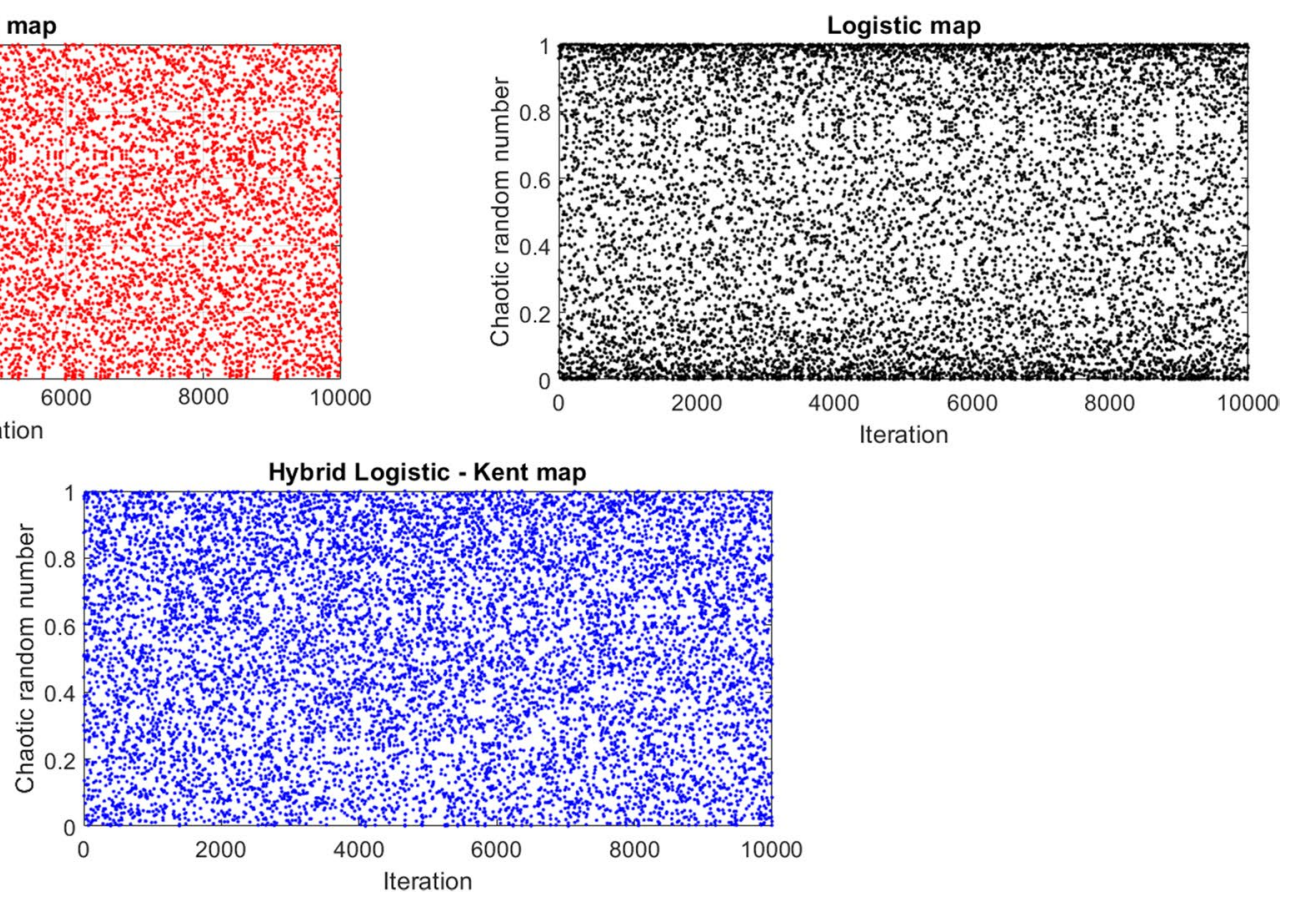

Fig. 1 Sequence of random numbers produced by Kent, Logistic, and the proposed hybrid chaotic map 
where $X$ stands for the current population; $V$ is the mutated $i^{\text {th }}$ population member; $r_{1}$ and $r_{2}$ are random integer indices defined in $[1, N]$; and $F$ is the scale factor that adjusts the scalar difference between two random population members $X_{r 2}$ and $X_{r 3}$. In this study, a constructive amelioration has been made on Eq. (3) by introducing an algorithm-specific iterative parameter par $_{\text {iter }}$ and onezero() function both of which are responsible for increasing the perturbation in the population. Equation (3) takes the below given final form defined Algorithm 3 by utilizing the mentioned functional amendments.

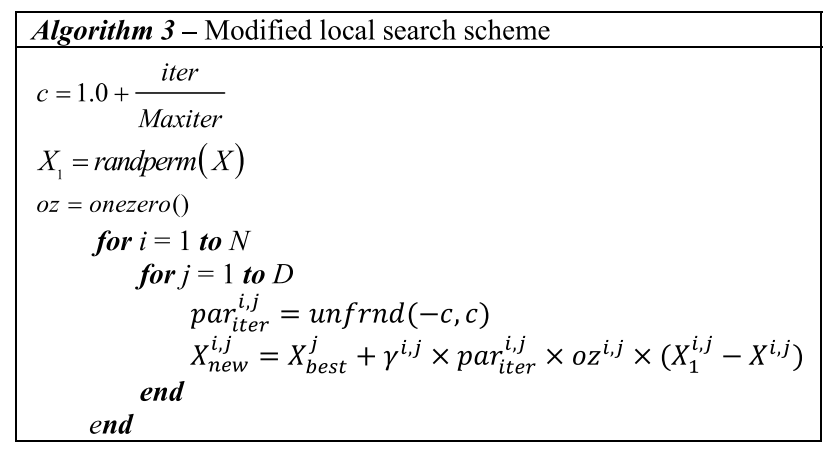

In Algorithm 3, iter is the current iteration whereas Maxiter represents the maximum number of iteration defining the termination criteria. Algorithm parameter $c$ is iteratively increased, which enables the current best solution $\left(X_{\text {best }}\right)$ to exploit the fertile regions occupying the neighborhood. The function unifrnd $(-c, c)$ creates a random number between $-c$ and $+c$ represented by the iteration dependent pariter parameter. This iterative parameter helps the algorithm to avoid local optimum points in the search space by refining the candidate solutions utilizing the promising areas nearby the current best solution. The perturbation function randperm() shuffles the row elements of the main population $(X)$ to produce a clone population $\left(X_{1}\right)$. The function onezero() generates $N \times D$ sized matrix composed of 1.0 or 0.0 valued integers and assigns each produced number to a population member. $\gamma$ is a chaotic random number defined in $[0,1]$ generated by Kent map as formulated in Eq. (1).

Figure 3 shows the variation of numerical values of the parameter par $_{\text {iter }}$ with an increasing number of iteration. As the respective formulation suggests, upper and lower bounds of the iterative values grow with the proceeding iterations which provides allowances for promising trial solutions to reach the unexplored spaces within the fertile search region. This parameter also avoids too much intensification on the current best solution to some extent, which sometimes results in getting trapped on some of the local best solutions leading to premature convergence. Based on the above-mentioned definitions and

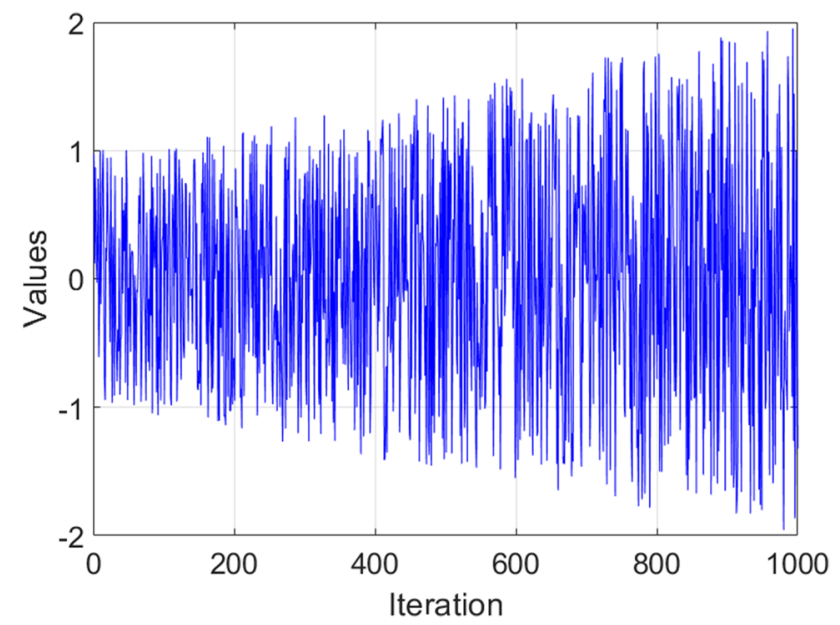

Fig. 3 Variation of the algorithm parameter par $_{\text {iter }}$ with the increasing number of iterations

formulations, the ensemble local search scheme takes the final form as given in Algorithm 4

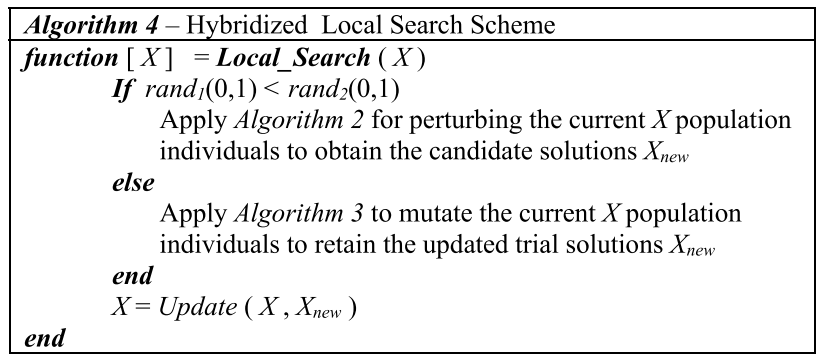

In Algorithm 4, rand $(0,1)$ and rand $_{2}(0,1)$ are uniformly distributed random numbers in $[0,1]$ which are also different from each other; $X$ is the current population whereas $X_{\text {new }}$ is the mutated population; Update() function compares the fitness quality of the updated and current solution vectors and copies the fittest individuals of $X_{\text {new }}$ into the $X$ current solution vector. The proposed algorithm inherits a generic structure which enables it to be implemented into various kind of available metaheuristic algorithms. To assess its applicability over various types of metaheuristic algorithms each of which having different structural characteristics, its successful implementation is considered into Crow search (CROW), Whale optimization (WHALE), differential evolution (DE), and sine cosine (SINECOS) algorithms. Next section deals with the performance evaluation of the algorithms in terms of the improvements made by the application of the proposed local search scheme. 


\section{Numerical experiments}

This section provides a comprehensive analysis of the influence of the proposed local search algorithm over the solution qualities of the metaheuristic algorithms. CROW algorithm is based on the pilfering behaviors of the intelligent crows. The WHALE optimization method simulates the characteristic social behavior of humpback whales. SINECOS algorithm utilizes the merits of sine and cosine functions to manipulate the trial solutions. DE is an evolutionary algorithm generating off-spring individuals by crossover, selection, and mutation operators. A test suite of forty optimization test functions composed of unimodal and multimodal benchmark problems are applied to these optimizers along with their local search improved versions. Unimodal test functions comprise only one global optimum point without involving any local optimum in the search domain. Therefore, they encourage the optimization algorithm to intensify on the promising regions which also allows for benchmarking the convergence speed and exploitation capabilities. Whereas, multimodal benchmark functions involve many local optimum points on the solution domain which makes them available and suitable for testing the algorithm performance regarding local minima avoidance and efficient exploration of the solution domain. 50 consecutive algorithm runs along with 100 maximum number of iterations are considered for each algorithm due to their inherent stochastic nature. For each test function to be solved, 20 search agents are employed. Among 50 different obtained solutions, the best of 30 runs have been considered for statistical analysis which is a reliable and quantitative performance measure for comparing the solution accuracy and consistency of the metaheuristic algorithms. Two different performance metrics including mean and standard deviation are put into practice to analyze convergence qualities of the compared algorithms. In this context, lower standard and mean deviation values indicate more robust and accurate solutions that verify the local minima avoidance and effective diversification of the search space. Table 1 reports the parameter configuration of the compared metaheuristic algorithms. Tables 2 and 3 provide a statistical analysis of the multimodal test functions. " $\mathrm{CL}$ " prefix in this context explains the assistance of the ensemble local search to the base metaheuristic method. For $f_{1}$-Levy test functions, it is seen that the embedded local search mechanism does not improve the quality of the solutions found by WHALE while other methods obtain slightly better solution thanks to the favorable contribution of the local search procedure. Local search boosted version of the compared
Table 1 Parameter configurations of the algorithms

\begin{tabular}{lll}
\hline Algorithm & Parameters & Value \\
\hline CROW & Flight length $(f l)$ & $f=2.0$ \\
& Awareness probability $(A P)$ & $A P=0.1$ \\
WHALE & Iterative parameter $(a)$ & $\begin{array}{c}a=\text { iteratively } \\
\text { decreasing } \\
\end{array}$ \\
& & from 2.0 to 0.0 \\
DE & Scale factor $(F)$ & $F=$ rand $(0,1)$ \\
& Crossover rate $(C R)$ & $C R=0.5$ \\
SINECOS & No algorithm parameters involved & - \\
\hline
\end{tabular}

metaheuristic algorithms acquire the global optimum results of $f_{2}$-Ackley, $f_{3}$-Griewank, $f_{4}$-Rastrigin, $f_{10}$-Schaffer, $f_{11}$-Inverted cosine, $f_{12}$-Wavy, $f_{14}$-Pathologic, $f_{17}$-Exponential, and $f_{22}$-Yang 4 for each algorithm run and proved its efficiency on multimodal test functions. As for the remaining test functions, the positive influence of the local search method on the respective solution accuracy and robustness is observed. Figures 4, 5, 6 and 7 visualize the convergence behaviors of the investigated metaheuristic algorithms. Berg and Engelbrecht [29] claim the idea that search agents should be effectively spread over a wide range of search domains at the initial phases of the iterations by the manipulation equations of the metaheuristic algorithm which enables to maintain an effective exploration and to obtain more diversified candidate solutions. As iterations proceed, extensive exploration is shifted into intensive exploitation which allows for intensification of the promising regions on the search space. Multimodal test functions are efficient testbeds for assessing the capabilities of the optimization algorithm regarding local minima avoidance. One can observe from Figs. 4 and 5 that implementation of the proposed hybrid local search scheme on metaheuristic algorithm entails a plausible balance between exploration and exploitation phases as in most of the convergence figures rapid changes occur at the initial steps then followed by sharp declines in the evolution of objective function. This convergence behavior explains that the search mechanism of the base algorithm is well-assisted by the proposed ensemble local search procedure as plenty of local optimum points are avoided during the iterations and the algorithm eventually convergences to a point in the solution, which is also in line with the standpoint of the claim pioneered by Berg and Engelbrecht [29]. Tables 4 and 5 report the error deviation results of the compared metaheuristics for unimodal test functions. It is seen that incorporating the ensemble local search scheme into the base algorithm 
Table 2 Statistical results of the multimodal test functions from $f_{1}$-Levy to $f_{16}$-Ackley N4

\begin{tabular}{|c|c|c|c|c|}
\hline & Mean \pm SD & Mean \pm SD & Mean \pm SD & Mean \pm SD \\
\hline & $f_{1}$-Levy & $f_{2}$-Ackley & $f_{3}$-Griewank & $f_{4}$-Rastrigin \\
\hline CROW & $2.93 \mathrm{E}+00 \pm 1.00 \mathrm{E}+00$ & $1.69 \mathrm{E}+00 \pm 2.56 \mathrm{E}-01$ & $3.01 \mathrm{E}-01 \pm 1.11 \mathrm{E}-01$ & $2.21 \mathrm{E}+02 \pm 2.17 \mathrm{E}+01$ \\
\hline CL-CROW & $4.58 \mathrm{E}-01 \pm 1.52 \mathrm{E}-01$ & $4.44 \mathrm{E}-16 \pm 0.00 \mathrm{E}+00$ & $0.00 \mathrm{E}+00 \pm 0.00 \mathrm{E}+00$ & $0.00 \mathrm{E}+00 \pm 0.00 \mathrm{E}+00$ \\
\hline WHALE & $1.37 \mathrm{E}+00 \pm 2.87 \mathrm{E}-01$ & $9.71 \mathrm{E}-07 \pm 1.26 \mathrm{E}-06$ & $3.78 \mathrm{E}-03 \pm 2.05 \mathrm{E}-02$ & $5.99 \mathrm{E}+00 \pm 2.71 \mathrm{E}+01$ \\
\hline CL-WHALE & $1.41 \mathrm{E}+00 \pm 3.51 \mathrm{E}-01$ & $4.44 E-16 \pm 0.00 E+00$ & $0.00 \mathrm{E}+00 \pm 0.00 \mathrm{E}+00$ & $0.00 \mathrm{E}+00 \pm 0.00 \mathrm{E}+00$ \\
\hline SINECOS & $2.21 \mathrm{E}+00 \pm 2.79 \mathrm{E}-01$ & $9.26 \mathrm{E}-02 \pm 6.99 \mathrm{E}-02$ & $2.22 \mathrm{E}-02 \pm 8.93 \mathrm{E}-02$ & $1.09 \mathrm{E}+02 \pm 5.05 \mathrm{E}+01$ \\
\hline CL-SINECOS & $2.03 \mathrm{E}+00 \pm 3.14 \mathrm{E}-01$ & $4.44 \mathrm{E}-16 \pm 0.00 \mathrm{E}+00$ & $0.00 \mathrm{E}+00 \pm 0.00 \mathrm{E}+00$ & $0.00 \mathrm{E}+00 \pm 0.00 \mathrm{E}+00$ \\
\hline $\mathrm{DE}$ & $2.01 \mathrm{E}+00 \pm 4.81 \mathrm{E}-01$ & $1.41 \mathrm{E}+00 \pm 1.67 \mathrm{E}-01$ & $2.95 \mathrm{E}-01 \pm 1.25 \mathrm{E}-01$ & $2.02 \mathrm{E}+02 \pm 1.32 \mathrm{E}+01$ \\
\hline \multirow[t]{2}{*}{ CL-DE } & $1.33 \mathrm{E}+00 \pm 2.87 \mathrm{E}-01$ & $4.44 E-16 \pm 0.00 E+00$ & $0.00 \mathrm{E}+00 \pm 0.00 \mathrm{E}+00$ & $0.00 \mathrm{E}+00 \pm 0.00 \mathrm{E}+00$ \\
\hline & $f_{5}$-Zakharov & $f_{6}$-Alpine & $f_{7}$-Penalized 1 & $f_{8}$-Quintic \\
\hline CROW & $1.01 \mathrm{E}+02 \pm 1.67 \mathrm{E}+01$ & $6.73 \mathrm{E}+00 \pm 1.91 \mathrm{E}+00$ & $9.55 \mathrm{E}-02 \pm 4.79 \mathrm{E}-02$ & $7.54 \mathrm{E}+02 \pm 5.17 \mathrm{E}+02$ \\
\hline CL-CROW & $9.66 \mathrm{E}-57 \pm 6.19 \mathrm{E}-56$ & $5.38 \mathrm{E}-41 \pm 1.73 \mathrm{E}-40$ & $5.11 \mathrm{E}-03 \pm 2.84 \mathrm{E}-03$ & $1.19 \mathrm{E}+01 \pm 5.09 \mathrm{E}+00$ \\
\hline WHALE & $9.13 \mathrm{E}+01 \pm 3.15 \mathrm{E}+01$ & $4.44 \mathrm{E}-02 \pm 3.44 \mathrm{E}-01$ & $6.57 \mathrm{E}-02 \pm 3.64 \mathrm{E}-02$ & $3.91 \mathrm{E}+01 \pm 1.02 \mathrm{E}+01$ \\
\hline CL-WHALE & $0.00 \mathrm{E}+00 \pm 0.00 \mathrm{E}+00$ & $0.00 \mathrm{E}+00 \pm 0.00 \mathrm{E}+00$ & $4.06 \mathrm{E}-02 \pm 2.45 \mathrm{E}-02$ & $2.97 \mathrm{E}+01 \pm 8.78 \mathrm{E}+01$ \\
\hline SINECOS & $5.43 \mathrm{E}+01 \pm 1.52 \mathrm{E}+01$ & $1.14 \mathrm{E}-01 \pm 2.11 \mathrm{E}-01$ & $3.21 \mathrm{E}-01 \pm 6.38 \mathrm{E}-02$ & $1.27 \mathrm{E}+02 \pm 6.61 \mathrm{E}+01$ \\
\hline CL-SINECOS & $0.00 \mathrm{E}+00 \pm 0.00 \mathrm{E}+00$ & $0.00 \mathrm{E}+00 \pm 0.00 \mathrm{E}+00$ & $1.88 \mathrm{E}-01 \pm 1.32 \mathrm{E}-01$ & $5.29 \mathrm{E}+01 \pm 9.30 \mathrm{E}+00$ \\
\hline $\mathrm{DE}$ & $1.19 \mathrm{E}+02 \pm 1.82 \mathrm{E}+01$ & $9.12 \mathrm{E}+00 \pm 1.82 \mathrm{E}+00$ & $2.24 \mathrm{E}-01 \pm 1.00 \mathrm{E}-02$ & $2.96 \mathrm{E}+02 \pm 1.58 \mathrm{E}+02$ \\
\hline \multirow[t]{2}{*}{ CL-DE } & $0.00 \mathrm{E}+00 \pm 0.00 \mathrm{E}+00$ & $0.00 \mathrm{E}+00 \pm 0.00 \mathrm{E}+00$ & $4.00 \mathrm{E}-01 \pm 2.22 \mathrm{E}-01$ & $4.95 \mathrm{E}+01 \pm 1.32 \mathrm{E}+01$ \\
\hline & $f_{9}$-Csendes & $f_{10}$-Schaffer & $f_{11}$-Inverted cosine & $f_{12}$-Wavy \\
\hline CROW & $9.01 \mathrm{E}+03 \pm 7.65 \mathrm{E}+03$ & $9.09 \mathrm{E}-02 \pm 1.21 \mathrm{E}-02$ & $2.72 \mathrm{E}+01 \pm 8.62 \mathrm{E}+00$ & $8.05 \mathrm{E}-01 \pm 2.49 \mathrm{E}-02$ \\
\hline CL-CROW & $8.85 \mathrm{E}-228 \pm 0.00 \mathrm{E}+00$ & $0.00 \mathrm{E}+00 \pm 0.00 \mathrm{E}+00$ & $0.00 \mathrm{E}+00 \pm 0.00 \mathrm{E}+00$ & $0.00 \mathrm{E}+00 \pm 0.00 \mathrm{E}+00$ \\
\hline WHALE & $4.19 \mathrm{E}-13 \pm 2.79 \mathrm{E}-12$ & $6.67 \mathrm{E}-03 \pm 5.96 \mathrm{E}-03$ & $2.96 \mathrm{E}-10 \pm 7.52 \mathrm{E}-10$ & $8.26 \mathrm{E}-02 \pm 1.87 \mathrm{E}-01$ \\
\hline CL-WHALE & $0.00 \mathrm{E}+00 \pm 0.00 \mathrm{E}+00$ & $0.00 \mathrm{E}+00 \pm 0.00 \mathrm{E}+00$ & $0.00 \mathrm{E}+00 \pm 0.00 \mathrm{E}+00$ & $0.00 \mathrm{E}+00 \pm 0.00 \mathrm{E}+00$ \\
\hline SINECOS & $6.91 \mathrm{E}+02 \pm 2.51 \mathrm{E}+03$ & $4.83 \mathrm{E}-02 \pm 1.34 \mathrm{E}-02$ & $5.97 \mathrm{E}-01 \pm 5.84 \mathrm{E}-01$ & $5.81 \mathrm{E}-01 \pm 1.21 \mathrm{E}-01$ \\
\hline CL-SINECOS & $0.00 \mathrm{E}+00 \pm 0.00 \mathrm{E}+00$ & $0.00 \mathrm{E}+00 \pm 0.00 \mathrm{E}+00$ & $0.00 \mathrm{E}+00 \pm 0.00 \mathrm{E}+00$ & $0.00 \mathrm{E}+00 \pm 0.00 \mathrm{E}+00$ \\
\hline $\mathrm{DE}$ & $4.43 \mathrm{E}+03 \pm 2.94 \mathrm{E}+03$ & $1.16 \mathrm{E}-01 \pm 1.25 \mathrm{E}-02$ & $1.97 \mathrm{E}+01 \pm 4.46 \mathrm{E}+00$ & $7.37 \mathrm{E}-01 \pm 2.39 \mathrm{E}-02$ \\
\hline \multirow[t]{2}{*}{ CL-DE } & $0.00 \mathrm{E}+00 \pm 0.00 \mathrm{E}+00$ & $0.00 \mathrm{E}+00 \pm 0.00 \mathrm{E}+00$ & $0.00 \mathrm{E}+00 \pm 0.00 \mathrm{E}+00$ & $0.00 \mathrm{E}+00 \pm 0.00 \mathrm{E}+00$ \\
\hline & $f_{13}$-Hyperellipsoid & $f_{14}$-Pathologic & $f_{15}$-Salomon & $f_{16}$-AckleyN4 \\
\hline CROW & $2.56 \mathrm{E}+03 \pm 8.63 \mathrm{E}+02$ & $4.89 \mathrm{E}+00 \pm 3.49 \mathrm{E}-01$ & $1.23 \mathrm{E}+00 \pm 1.13 \mathrm{E}-01$ & $-4.98 \mathrm{E}-02 \pm 1.47 \mathrm{E}+01$ \\
\hline CL-CROW & $6.17 \mathrm{E}-77 \pm 2.54 \mathrm{E}-76$ & $0.00 \mathrm{E}+00 \pm 0.00 \mathrm{E}+00$ & $2.15 \mathrm{E}-37 \pm 1.95 \mathrm{E}-36$ & $-6.42 \mathrm{E}+01 \pm 9.75 \mathrm{E}+00$ \\
\hline WHALE & $2.62 \mathrm{E}-08 \pm 1.35 \mathrm{E}-07$ & $3.89 \mathrm{E}+00 \pm 8.26 \mathrm{E}-01$ & $1.81 \mathrm{E}-01 \pm 1.14 \mathrm{E}-01$ & $-2.18 \mathrm{E}+01 \pm 2.01 \mathrm{E}+01$ \\
\hline CL-WHALE & $0.00 \mathrm{E}+00 \pm 0.00 \mathrm{E}+00$ & $0.00 \mathrm{E}+00 \pm 0.00 \mathrm{E}+00$ & $0.00 \mathrm{E}+00 \pm 0.00 \mathrm{E}+00$ & $-5.43 \mathrm{E}+01 \pm 1.10 \mathrm{E}+01$ \\
\hline SINECOS & $1.20 \mathrm{E}+01 \pm 2.32 \mathrm{E}+01$ & $4.79 \mathrm{E}+00 \pm 3.35 \mathrm{E}-01$ & $6.56 \mathrm{E}-01 \pm 1.78 \mathrm{E}-01$ & $3.80 \mathrm{E}+00 \pm 1.00 \mathrm{E}+01$ \\
\hline CL-SINECOS & $0.00 \mathrm{E}+00 \pm 0.00 \mathrm{E}+00$ & $0.00 \mathrm{E}+00 \pm 0.00 \mathrm{E}+00$ & $0.00 \mathrm{E}+00 \pm 0.00 \mathrm{E}+00$ & $-3.36 \mathrm{E}+01 \pm 1.12 \mathrm{E}+01$ \\
\hline $\mathrm{DE}$ & $1.98 \mathrm{E}+03 \pm 6.09 \mathrm{E}+02$ & $3.96 \mathrm{E}+00 \pm 3.17 \mathrm{E}-01$ & $1.40 \mathrm{E}+00 \pm 1.06 \mathrm{E}-01$ & $-3.10 \mathrm{E}-01 \pm 9.15 \mathrm{E}+00$ \\
\hline CL-DE & $0.00 \mathrm{E}+00 \pm 0.00 \mathrm{E}+00$ & $0.00 E+00 \pm 0.00 E+00$ & $0.00 E+00 \pm 0.00 E+00$ & $-4.92 \mathrm{E}+01 \pm 1.13 \mathrm{E}+01$ \\
\hline
\end{tabular}

Bold values are the best solutions obtained by the corresponding optimization algorithm for the related problem

enormously improves the solution quality as the global optimum solution is obtained for each algorithm run in most of the test cases except for the CL-CROW optimizer. The global optimum solution of $f_{24}$-Rosenbrock, $f_{33}$-DixonPrice, and $f_{39}$-Schwefel 2.25 test functions are not acquired by any of the algorithm reported in Tables 4 and 5 . However, there is a significant gap between the optimal results found by the local search improved and base algorithms. Besides, amelioration in the solution quality through the utilization of the local search mechanism is observably evident for these test functions. Figures 8, 9 and 10 depict the evolution characteristics of the fitness functions of each optimization algorithm compared in Tables 4 and 5. The quick and rapid convergence of the global optimum point is observed from the figures almost for each benchmark function. Acceleration in convergence speed is obvious and observable particularly after half of the iterations which emphasizes the fact the hybrid algorithm 
Table 3 Statistical results for multimodal benchmark functions from $f_{17}$-Exponential to $f_{22}$-Yang 4

\begin{tabular}{|c|c|c|c|}
\hline & Mean \pm SD & Mean \pm SD & Mean \pm SD \\
\hline & $f_{17}$-Exponential & $f_{18}$-Trid 6 & $f_{19}$-Styblinski-Tang \\
\hline CROW & $-3.00 \mathrm{E}-04 \pm 9.87 \mathrm{E}-04$ & $3.13 \mathrm{E}+05 \pm 1.13 \mathrm{E}+05$ & $-6.86 \mathrm{E}+02 \pm 6.90 \mathrm{E}+01$ \\
\hline CL-CROW & $-1.00 \mathrm{E}+00 \pm 0.00 \mathrm{E}+00$ & $-2.18 \mathrm{E}+02 \pm 3.60 \mathrm{E}+01$ & $-9.61 \mathrm{E}+02 \pm 4.94 \mathrm{E}+01$ \\
\hline WHALE & $9.99 \mathrm{E}-01 \pm 1.21 \mathrm{E}-10$ & $-1.51 \mathrm{E}+02 \pm 1.27 \mathrm{E}+02$ & $-6.81 \mathrm{E}+02 \pm 9.77 \mathrm{E}+01$ \\
\hline CL-WHALE & $-1.00 \mathrm{E}+00 \pm 0.00 \mathrm{E}+00$ & $-2.69 \mathrm{E}+02 \pm 6.28 \mathrm{E}+01$ & $-8.54 \mathrm{E}+02 \pm 7.98 \mathrm{E}+01$ \\
\hline SINECOS & $-9.17 \mathrm{E}-01 \pm 1.27 \mathrm{E}-01$ & $-1.81 \mathrm{E}+01 \pm 1.51 \mathrm{E}+01$ & $-4.69 \mathrm{E}+02 \pm 4.01 \mathrm{E}+01$ \\
\hline CL-SINECOS & $-1.00 E+00 \pm 0.00 E+00$ & $-6.90 \mathrm{E}+01 \pm 3.67 \mathrm{E}+01$ & $-7.36 \mathrm{E}+02 \pm 7.25 \mathrm{E}+02$ \\
\hline DE & $-1.61 \mathrm{E}-03 \pm 4.97 \mathrm{E}-03$ & $-3.65 E+02 \pm 1.87 E+01$ & $-6.67 \mathrm{E}+02 \pm 3.21 \mathrm{E}+01$ \\
\hline \multirow[t]{2}{*}{ CL-DE } & $-1.00 \mathrm{E}+00 \pm 0.00 \mathrm{E}+00$ & $-3.56 \mathrm{E}+02 \pm 3.98 \mathrm{E}+01$ & $-7.53 \mathrm{E}+02 \pm 6.38 \mathrm{E}+01$ \\
\hline & $f_{20}$-Yang1 & $f_{21}$-Yang2 & $f_{22}$-Yang4 \\
\hline CROW & $1.20 \mathrm{E}+08 \pm 3.47 \mathrm{E}+08$ & $2.26 \mathrm{E}-05 \pm 2.12 \mathrm{E}-05$ & $1.31 \mathrm{E}-11 \pm 7.70 \mathrm{E}-12$ \\
\hline CL-CROW & $1.34 \mathrm{E}-63 \pm 5.91 \mathrm{E}-63$ & $8.73 \mathrm{E}-07 \pm 1.31 \mathrm{E}-06$ & $-1.00 \mathrm{E}+00 \pm 0.00 \mathrm{E}+00$ \\
\hline WHALE & $3.38 \mathrm{E}+12 \pm 3.18 \mathrm{E}+13$ & $9.95 \mathrm{E}-06 \pm 2.86 \mathrm{E}-05$ & $1.73 \mathrm{E}-11 \pm 1.05 \mathrm{E}-11$ \\
\hline CL-WHALE & $0.00 \mathrm{E}+00 \pm 0.00 \mathrm{E}+00$ & $3.90 \mathrm{E}-06 \pm 1.17 \mathrm{E}-05$ & $-1.00 E+00 \pm 0.00 E+00$ \\
\hline SINECOS & $2.86 \mathrm{E}+05 \pm 2.32 \mathrm{E}+06$ & $1.30 \mathrm{E}-05 \pm 1.49 \mathrm{E}-05$ & $1.08 \mathrm{E}-10 \pm 5.50 \mathrm{E}-11$ \\
\hline CL-SINECOS & $0.00 \mathrm{E}+00 \pm 0.00 \mathrm{E}+00$ & $4.57 E-10 \pm 6.18 E-10$ & $-1.00 E+00 \pm 0.00 E+00$ \\
\hline DE & $5.74 \mathrm{E}+07 \pm 2.94 \mathrm{E}+08$ & $1.40 \mathrm{E}-06 \pm 1.31 \mathrm{E}-06$ & $1.01 \mathrm{E}-11 \pm 3.62 \mathrm{E}-12$ \\
\hline CL-DE & $0.00 \mathrm{E}+00 \pm 0.00 \mathrm{E}+00$ & $3.78 \mathrm{E}-08 \pm 5.78 \mathrm{E}-08$ & $-1.00 \mathrm{E}+00 \pm 0.00 \mathrm{E}+00$ \\
\hline
\end{tabular}

Bold values are the best solutions obtained by the corresponding optimization algorithm for the related problem

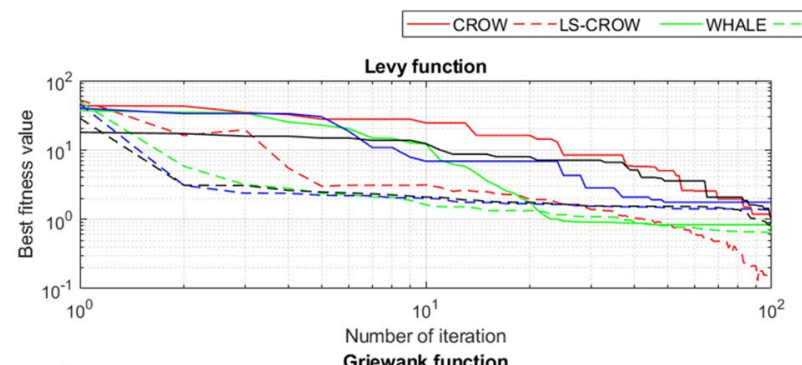
- LS-WHALE
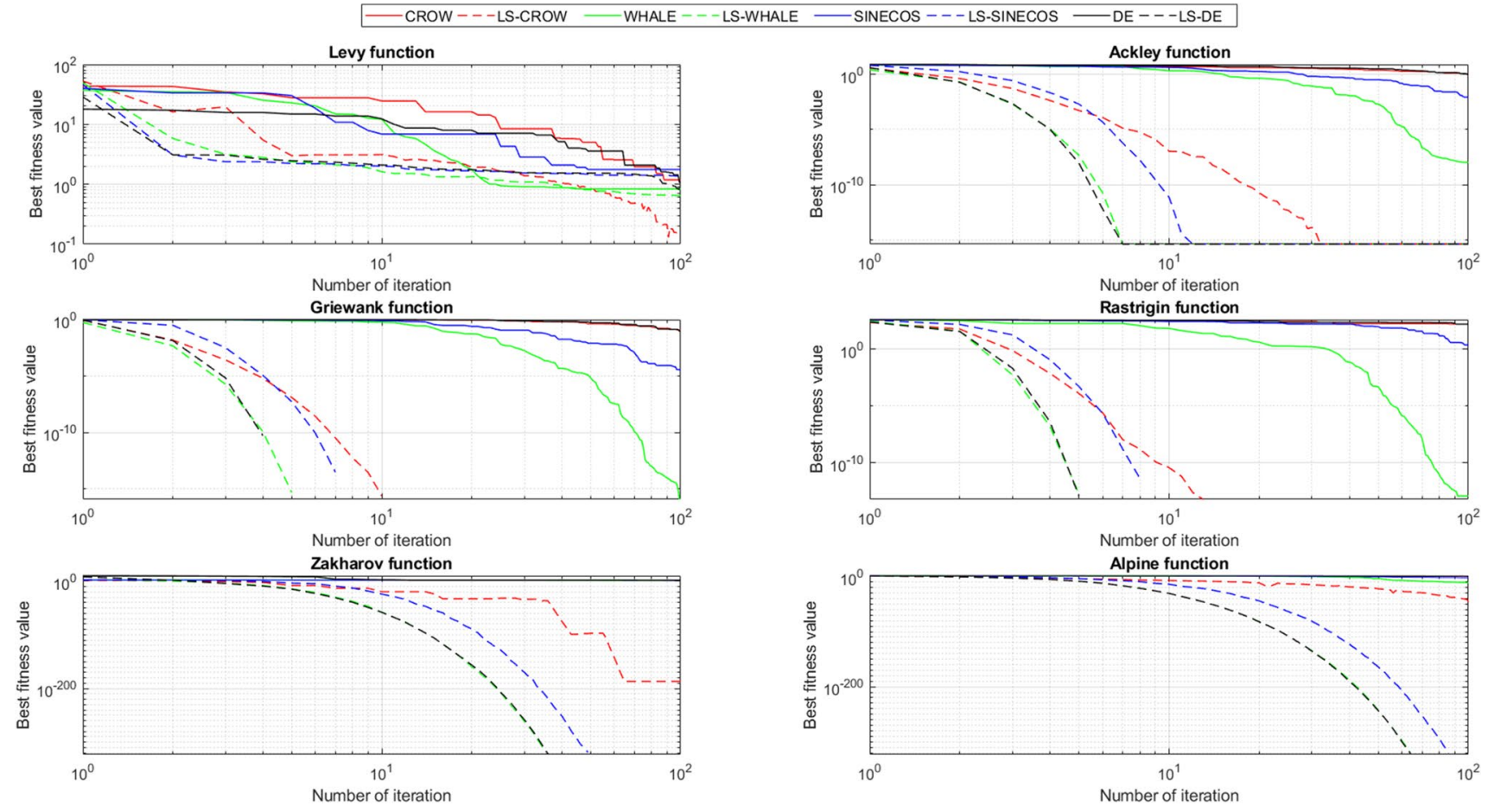

Fig. 4 Evolution histories of the compared algorithms for benchmark functions from $f_{1}$-Levy to $f_{6}$-Alpine

implements the exploitation phase polishing the so-farobtained best solutions by moving around the fertile and promising regions. It can be deduced from these conclusive findings and discussions that avoiding local optima in the search space and quick convergence to the global optimum solution are two prominent advantages provided by the integration of the generic local search algorithm into sample metaheuristic algorithms. 


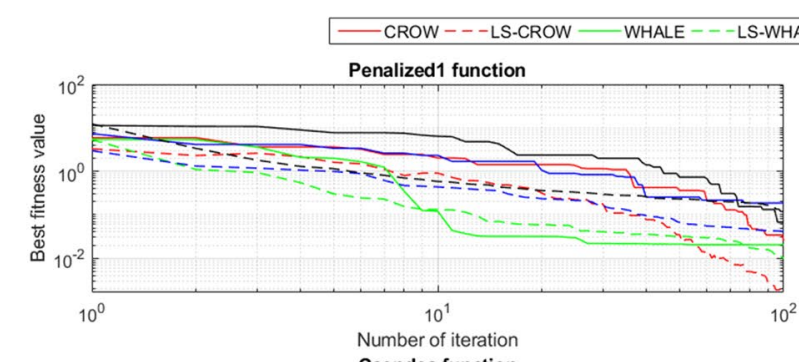

- SINECOS ---LS-SINECOS - DE --- LS-DE
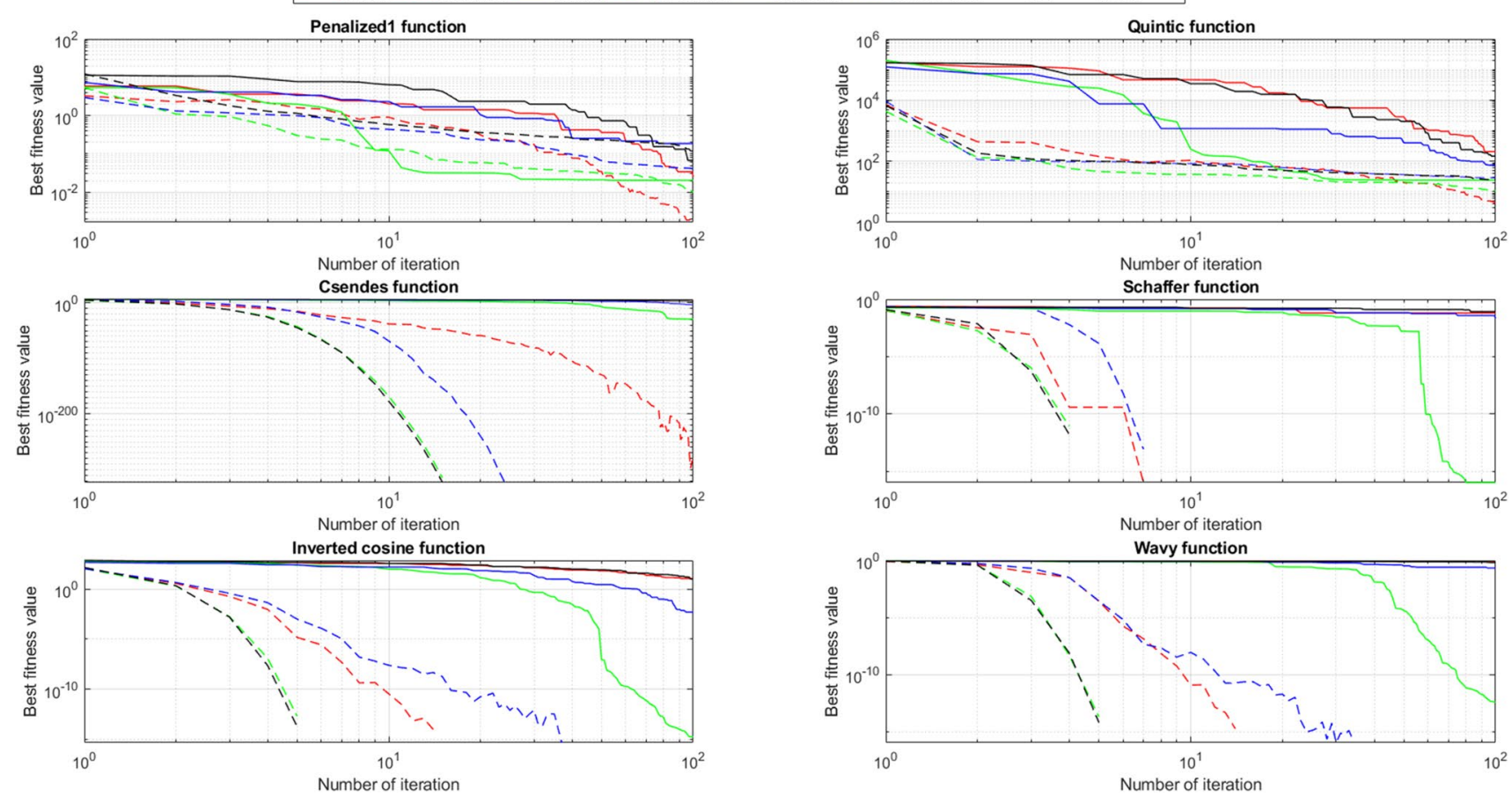

Fig. 5 Convergence characteristics of the metaheuristic algorithms for test functions from $f_{7}$-Penalized 1 to $f_{12}$-Wavy test functions
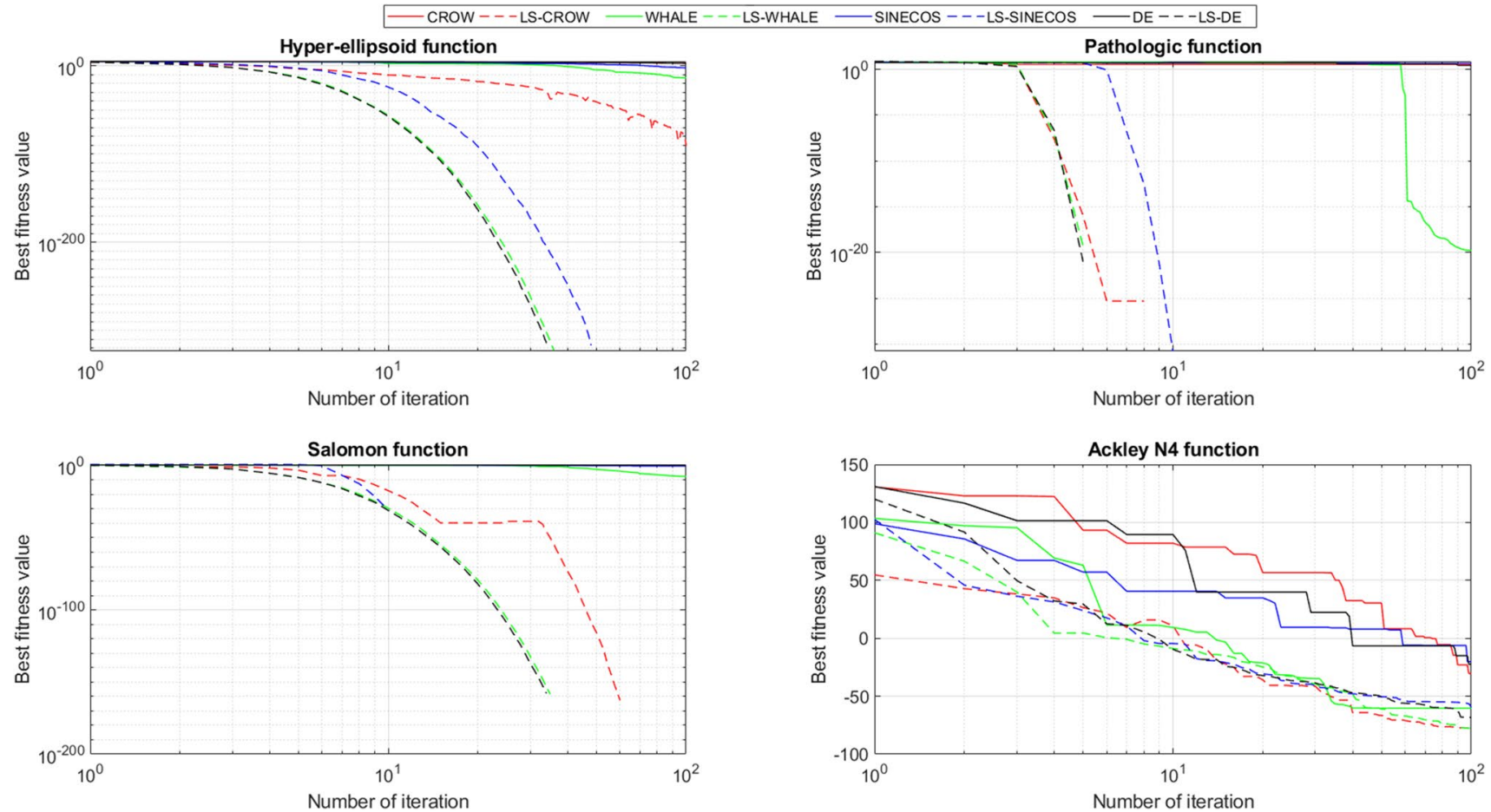

Fig. 6 Convergence histories of the compared algorithms for test functions from $f_{13}$-Hyperellipsoid to $f_{16}$-Ackley N4 


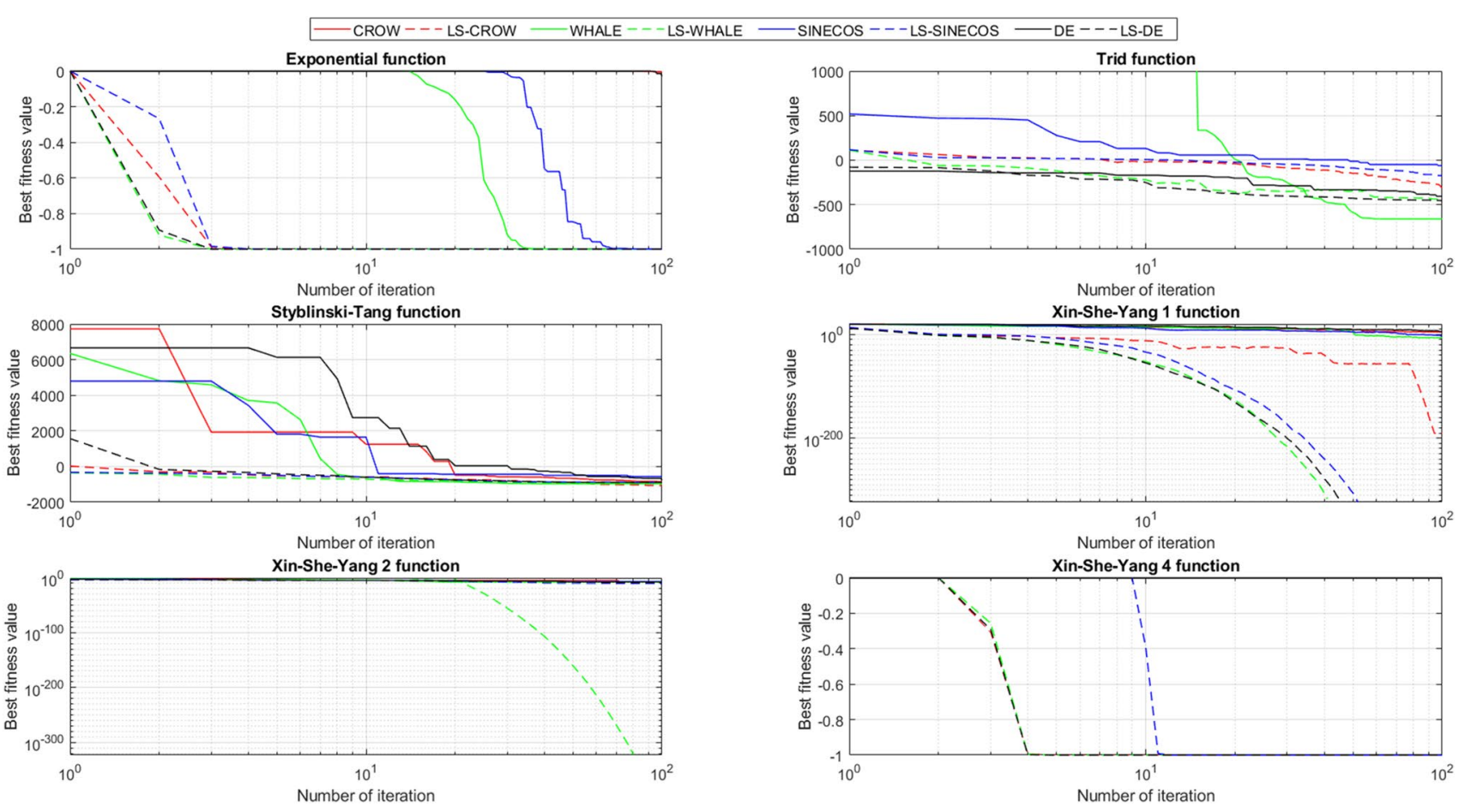

Fig. 7 Evolution characteristics of the metaheuristic algorithms for test functions from $f_{17}$-Exponential to $f_{22}$-Yang 4

\section{Application of the local search improved algorithms on engineering design problems}

Despite the significant success shown in unconstrained multidimensional benchmark problems, an exhaustive analysis of their efficiency will be assessed on real-world constrained optimization problems with having challenging constraints. To integrate the imposed design constraints on the objective function, a very simple yet effective death penalty method is taken into account whose mathematical formulation will be expressed below. By using the static penalty method, a constrained design problem is converted into an unconstrained optimization problem by penalizing the solutions violating the prescribed constraints. Eliminating an unfeasible solution on the search space can be maintained by the following equation the number of inequality and equality constraints over the solution domain. Parameters penal 1 and penal $_{2}$ are high-valued numbers that correspondingly penalize the unfeasible solutions violating the equality and inequality constraint functions. Parameters $\sigma$ and $\rho$ are respectively considered to be 1.0 and 2.0. Two challenging real-world optimization cases with binding constraints including optimal design of a refrigeration system and optimal design of a heat exchanger network problems will be solved by the above-mentioned metaheuristic algorithms. For each algorithm, 20 search agents with a maximum number of 10,000 iterations are considered. Due to the inherent stochastic nature of the metaheuristic algorithms, the best 30 out of 50 solutions obtained by the compared methods from the consecutive runs are compiled for the statistical analysis.

$f(\vec{x})=f_{o b j}(\vec{x}) \pm\left[\sum_{i=1}^{M}\right.$ penal $_{1, i} \cdot \max \left(0, g_{i}(\vec{x})\right)^{\sigma}+\sum_{j=1}^{N}$ penal $\left._{2, j} \cdot\left|h_{j}(\vec{x})\right|^{\rho}\right]$

where $f(\vec{x})$ is the penalized objective function and $f_{o b j}(\vec{x})$ is the pure objective function of the optimization problem. Functions $g(\vec{x})$ and $h(\vec{x})$ respectively symbolize the inequality and equality constraints defined for the specific optimization problem. $M$ and $N$ respectively represent

\subsection{Optimal design of a refrigeration system}

The mathematical model defined in Pant et al. [30] for optimizing the system efficiency can be expressed by the following set of equations 
Table 4 Deviation results for unimodal test function from $f_{23}$-Sphere to $f_{34}$-Yang 3

\begin{tabular}{|c|c|c|c|c|}
\hline & Mean \pm SD & Mean \pm SD & Mean \pm SD & Mean \pm SD \\
\hline & $f_{23}$-Sphere & $f_{24}$-Rosenbrock & $f_{25}$-Brown & $f_{26}$-Streched sine wave \\
\hline CROW & $2.29 \mathrm{E}+02 \pm 6.16 \mathrm{E}+00$ & $2.58 \mathrm{E}+04 \pm 1.40 \mathrm{E}+\mathrm{E}+04$ & $6.29 \mathrm{E}+11 \pm 5.47 \mathrm{E}+12$ & $4.41 \mathrm{E}+01 \pm 3.98 \mathrm{E}+00$ \\
\hline CL-CROW & $2.04 \mathrm{E}-78 \pm 8.94 \mathrm{E}-78$ & $2.83 \mathrm{E}+01 \pm 2.65 \mathrm{E}-01$ & $9.75 \mathrm{E}-77 \pm 8.15 \mathrm{E}-76$ & $5.81 \mathrm{E}-20 \pm 6.54 \mathrm{E}-20$ \\
\hline WHALE & $3.72 \mathrm{E}-11 \pm 9.54 \mathrm{E}-11$ & $2.88 \mathrm{E}+01 \pm 7.85 \mathrm{E}-01$ & $3.15 \mathrm{E}-12 \pm 7.30 \mathrm{E}-12$ & $3.10 \mathrm{E}-03 \pm 1.21 \mathrm{E}-02$ \\
\hline CL-WHALE & $0.00 \mathrm{E}+00 \pm 0.00 \mathrm{E}+00$ & $2.87 \mathrm{E}+01 \pm 1.74 \mathrm{E}-01$ & $0.00 \mathrm{E}+00 \pm 0.00 \mathrm{E}+00$ & $0.00 \mathrm{E}+00 \pm 0.00 \mathrm{E}+00$ \\
\hline SINECOS & $1.53 \mathrm{E}-01 \pm 3.37 \mathrm{E}-01$ & $4.95 \mathrm{E}+02 \pm 1.06 \mathrm{E}+03$ & $1.16 \mathrm{E}+00 \pm 2.54 \mathrm{E}+00$ & $8.37 \mathrm{E}+00 \pm 3.28 \mathrm{E}+00$ \\
\hline CL-SINECOS & $9.28 \mathrm{E}-31 \pm 4.25 \mathrm{E}-30$ & $2.86 \mathrm{E}+01 \pm 1.97 \mathrm{E}-01$ & $0.00 E+00 \pm 0.00 E+00$ & $0.00 \mathrm{E}+00 \pm 0.00 \mathrm{E}+00$ \\
\hline $\mathrm{DE}$ & $1.69 \mathrm{E}+01 \pm 4.11 \mathrm{E}+00$ & $1.46 \mathrm{E}+04 \pm 6.54 \mathrm{E}+03$ & $2.06 \mathrm{E}+20 \pm 1.93 \mathrm{E}+21$ & $4.63 \mathrm{E}+01 \pm 3.61 \mathrm{E}+00$ \\
\hline \multirow[t]{2}{*}{ CL-DE } & $0.00 \mathrm{E}+00 \pm 0.00 \mathrm{E}+00$ & $2.84 \mathrm{E}+01 \pm 2.25 \mathrm{E}-01$ & $0.00 \mathrm{E}+00 \pm 0.00 \mathrm{E}+00$ & $0.00 \mathrm{E}+00 \pm 0.00 \mathrm{E}+00$ \\
\hline & $f_{27}$-Powell singular & $f_{28}$-Sum of different powers & $f_{29}$-Sum of squares & $f_{30}$-Bent cigar \\
\hline CROW & $8.26 \mathrm{E}+03 \pm 1.54 \mathrm{E}+04$ & $7.70 \mathrm{E}+07 \pm 3.41 \mathrm{E}+08$ & $2.75 \mathrm{E}+02 \pm 7.44 \mathrm{E}+01$ & $2.24 \mathrm{E}+07 \pm 8.93 \mathrm{E}+06$ \\
\hline CL-CROW & $9.74 \mathrm{E}-76 \pm 6.21 \mathrm{E}-75$ & $1.53 \mathrm{E}-51 \pm 1.18 \mathrm{E}-50$ & $4.23 \mathrm{E}-77 \pm 1.32 \mathrm{E}-76$ & $5.18 \mathrm{E}-72 \pm 2.69 \mathrm{E}-71$ \\
\hline WHALE & $2.46 \mathrm{E}-09 \pm 6.74 \mathrm{E}-09$ & $8.83 \mathrm{E}-03 \pm 6.32 \mathrm{E}-02$ & $7.61 \mathrm{E}-10 \pm 2.19 \mathrm{E}-09$ & $2.10 \mathrm{E}-05 \pm 5.57 \mathrm{E}-05$ \\
\hline CL-WHALE & $0.00 \mathrm{E}+00 \pm 0.00 \mathrm{E}+00$ & $0.00 \mathrm{E}+00 \pm 0.00 \mathrm{E}+00$ & $0.00 \mathrm{E}+00 \pm 0.00 \mathrm{E}+00$ & $0.00 \mathrm{E}+00 \pm 0.00 \mathrm{E}+00$ \\
\hline SINECOS & $1.35 \mathrm{E}+02 \pm 2.62 \mathrm{E}+02$ & $8.69 \mathrm{E}+04 \pm 6.48 \mathrm{E}+05$ & $1.29 \mathrm{E}+00 \pm 1.77 \mathrm{E}+00$ & $9.40 \mathrm{E}+04 \pm 1.36 \mathrm{E}+05$ \\
\hline CL-SINECOS & $0.00 \mathrm{E}+00 \pm 0.00 \mathrm{E}+00$ & $2.22 \mathrm{E}-33 \pm 1.84 \mathrm{E}-32$ & $0.00 \mathrm{E}+00 \pm 0.00 \mathrm{E}+00$ & $0.00 E+00 \pm 0.00 E+00$ \\
\hline $\mathrm{DE}$ & $2.31 \mathrm{E}+04 \pm 6.81 \mathrm{E}+03$ & $5.25 \mathrm{E}+06 \pm 1.34 \mathrm{E}+07$ & $1.96 \mathrm{E}+01 \pm 4.59 \mathrm{E}+01$ & $1.42 \mathrm{E}+07 \pm 3.95 \mathrm{E}+06$ \\
\hline \multirow[t]{2}{*}{ CL-DE } & $0.00 \mathrm{E}+00 \pm 0.00 \mathrm{E}+00$ & $0.00 \mathrm{E}+00 \pm 0.00 \mathrm{E}+00$ & $0.00 \mathrm{E}+00 \pm 0.00 \mathrm{E}+00$ & $0.00 \mathrm{E}+00 \pm 0.00 \mathrm{E}+00$ \\
\hline & $f_{31}$-Discus & $f_{32}$-Different powers & $f_{33}$-Dixon-Price & $f_{34}$-Yang 3 \\
\hline CROW & $3.18 \mathrm{E}+01 \pm 7.86 \mathrm{E}+00$ & $9.51 \mathrm{E}+00 \pm 2.13 \mathrm{E}+00$ & $9.90 \mathrm{E}+11 \pm 5.79 \mathrm{E}+11$ & $8.91 \mathrm{E}-01 \pm 8.71 \mathrm{E}-03$ \\
\hline CL-CROW & $7.56 \mathrm{E}-78 \pm 3.26 \mathrm{E}-77$ & $5.77 \mathrm{E}-49 \pm 1.86 \mathrm{E}-48$ & $6.91 \mathrm{E}-01 \pm 7.04 \mathrm{E}-02$ & $-1.00 \mathrm{E}+00 \pm 0.00 \mathrm{E}+00$ \\
\hline WHALE & $3.72 \mathrm{E}-10 \pm 1.41 \mathrm{E}-09$ & $2.01 \mathrm{E}-06 \pm 7.64 \mathrm{E}-06$ & $9.81 \mathrm{E}-01 \pm 4.83 \mathrm{E}-02$ & $8.86 \mathrm{E}-01 \pm 1.49 \mathrm{E}-02$ \\
\hline CL-WHALE & $0.00 \mathrm{E}+00 \pm 0.00 \mathrm{E}+00$ & $0.00 \mathrm{E}+00 \pm 0.00 \mathrm{E}+00$ & $6.81 \mathrm{E}-01 \pm 6.11 \mathrm{E}-01$ & $-1.00 \mathrm{E}+00 \pm 0.00 \mathrm{E}+00$ \\
\hline SINECOS & $1.78 \mathrm{E}-01 \pm 2.57 \mathrm{E}-01$ & $7.21 \mathrm{E}-01 \pm 8.75 \mathrm{E}-01$ & $8.57 \mathrm{E}+09 \pm 2.66 \mathrm{E}+10$ & $9.03 \mathrm{E}-01 \pm 7.72 \mathrm{E}-03$ \\
\hline CL-SINECOS & $0.00 \mathrm{E}+00 \pm 0.00 \mathrm{E}+00$ & $0.00 \mathrm{E}+00 \pm 0.00 \mathrm{E}+00$ & $7.15 \mathrm{E}-01 \pm 8.85 \mathrm{E}-02$ & $-9.99 \mathrm{E}-01 \pm 1.09 \mathrm{E}-06$ \\
\hline $\mathrm{DE}$ & $3.14 \mathrm{E}+01 \pm 6.97 \mathrm{E}+00$ & $8.14 \mathrm{E}+00 \pm 1.72 \mathrm{E}+00$ & $4.89 \mathrm{E}+11 \pm 2.26 \mathrm{E}+11$ & $8.31 \mathrm{E}-01 \pm 9.65 \mathrm{E}-03$ \\
\hline CL-DE & $0.00 \mathrm{E}+00 \pm 0.00 \mathrm{E}+00$ & $0.00 \mathrm{E}+00 \pm 0.00 \mathrm{E}+00$ & $6.66 \mathrm{E}-01 \pm 8.39 \mathrm{E}-01$ & $-1.00 \mathrm{E}+00 \pm 0.00 \mathrm{E}+00$ \\
\hline
\end{tabular}

Bold values are the best solutions obtained by the corresponding optimization algorithm for the related problem

Table 5 Error analysis for unimodal benchmark functions from $f_{35}$-Schwefel 2.20 to $f_{40}$-Dropwave

\begin{tabular}{|c|c|c|c|}
\hline & Mean \pm SD & Mean \pm SD & Mean \pm SD \\
\hline & $f_{35}$-Schwefel 2.20 & $f_{36}$-Schwefel 2.21 & $f_{37}$-Schwefel 2.22 \\
\hline CROW & $1.51 \mathrm{E}+01 \pm 2.76 \mathrm{E}+00$ & $3.32 \mathrm{E}-18 \pm 1.21 \mathrm{E}-17$ & $1.59 \mathrm{E}+01 \pm 3.08 \mathrm{E}+00$ \\
\hline CL-CROW & $6.39 \mathrm{E}-40 \pm 1.36 \mathrm{E}-39$ & $4.53 \mathrm{E}-52 \pm 3.27 \mathrm{E}-52$ & $1.95 \mathrm{E}-39 \pm 4.61 \mathrm{E}-39$ \\
\hline WHALE & $1.02 \mathrm{E}-07 \pm 2.53 \mathrm{E}-07$ & $1.08 \mathrm{E}-36 \pm 5.27 \mathrm{E}-36$ & $5.40 \mathrm{E}-08 \pm 1.25 \mathrm{E}-07$ \\
\hline CL-WHALE & $0.00 \mathrm{E}+00 \pm 0.00 \mathrm{E}+00$ & $0.00 E+00 \pm 0.00 E+00$ & $0.00 \mathrm{E}+00 \pm 0.00 \mathrm{E}+00$ \\
\hline SINECOS & $1.19 \mathrm{E}-01 \pm 9.81 \mathrm{E}-02$ & $7.97 \mathrm{E}-29 \pm 4.43 \mathrm{E}-28$ & $1.26 \mathrm{E}-01 \pm 9.70 \mathrm{E}-02$ \\
\hline CL-SINECOS & $9.42 \mathrm{E}-19 \pm 5.37 \mathrm{E}-18$ & $0.00 \mathrm{E}+00 \pm 0.00 \mathrm{E}+00$ & $0.00 \mathrm{E}+00 \pm 0.00 \mathrm{E}+00$ \\
\hline DE & $1.32 \mathrm{E}+01 \pm 2.13 \mathrm{E}+00$ & $3.45 \mathrm{E}-12 \pm 6.47 \mathrm{E}-12$ & $1.50 \mathrm{E}+01 \pm 2.43 \mathrm{E}+00$ \\
\hline \multirow[t]{2}{*}{ CL-DE } & $0.00 \mathrm{E}+00 \pm 0.00 \mathrm{E}+00$ & $0.00 \mathrm{E}+00 \pm 0.00 \mathrm{E}+00$ & $0.00 \mathrm{E}+00 \pm 0.00 \mathrm{E}+00$ \\
\hline & $f_{38}$-Schwefel 2.23 & $f_{39}$-Schwefel 2.25 & $f_{40}$-Dropwave \\
\hline CROW & $1.28 \mathrm{E}+06 \pm 1.21 \mathrm{E}+06$ & $3.92 \mathrm{E}+02 \pm 1.31 \mathrm{E}+02$ & $-3.29 \mathrm{E}-02 \pm 8.56 \mathrm{E}-03$ \\
\hline CL-CROW & $0.00 \mathrm{E}+00 \pm 0.00 \mathrm{E}+00$ & $3.98 \mathrm{E}+00 \pm 1.03 \mathrm{E}+00$ & $-9.36 \mathrm{E}-01 \pm 1.90 \mathrm{E}-05$ \\
\hline WHALE & $5.48 \mathrm{E}-09 \pm 4.42 \mathrm{E}-08$ & $1.32 \mathrm{E}+01 \pm 3.21 \mathrm{E}+00$ & $-8.61 \mathrm{E}-01 \pm 1.14 \mathrm{E}-01$ \\
\hline CL-WHALE & $0.00 \mathrm{E}+00 \pm 0.00 \mathrm{E}+00$ & $7.89 \mathrm{E}+00 \pm 2.58 \mathrm{E}+00$ & $-1.00 E+00 \pm 0.00 E+00$ \\
\hline SINECOS & $2.54 \mathrm{E}+05 \pm 6.91 \mathrm{E}+05$ & $6.06 \mathrm{E}+01 \pm 8.39 \mathrm{E}+01$ & $-2.55 \mathrm{E}-01 \pm 1.44 \mathrm{E}-01$ \\
\hline CL-SINECOS & $0.00 \mathrm{E}+00 \pm 0.00 \mathrm{E}+00$ & $1.69 \mathrm{E}+01 \pm 1.67 \mathrm{E}+00$ & $-1.00 E+00 \pm 0.00 E+00$ \\
\hline DE & $5.34 \mathrm{E}+05 \pm 5.57 \mathrm{E}+05$ & $4.47 \mathrm{E}+02 \pm 1.73 \mathrm{E}+02$ & $-2.54 \mathrm{E}-02 \pm 4.98 \mathrm{E}-03$ \\
\hline CL-DE & $0.00 \mathrm{E}+00 \pm 0.00 \mathrm{E}+00$ & $1.34 \mathrm{E}+01 \pm 2.27 \mathrm{E}+00$ & $-1.00 \mathrm{E}+00 \pm 0.00 \mathrm{E}+00$ \\
\hline
\end{tabular}

Bold values are the best solutions obtained by the corresponding optimization algorithm for the related problem 

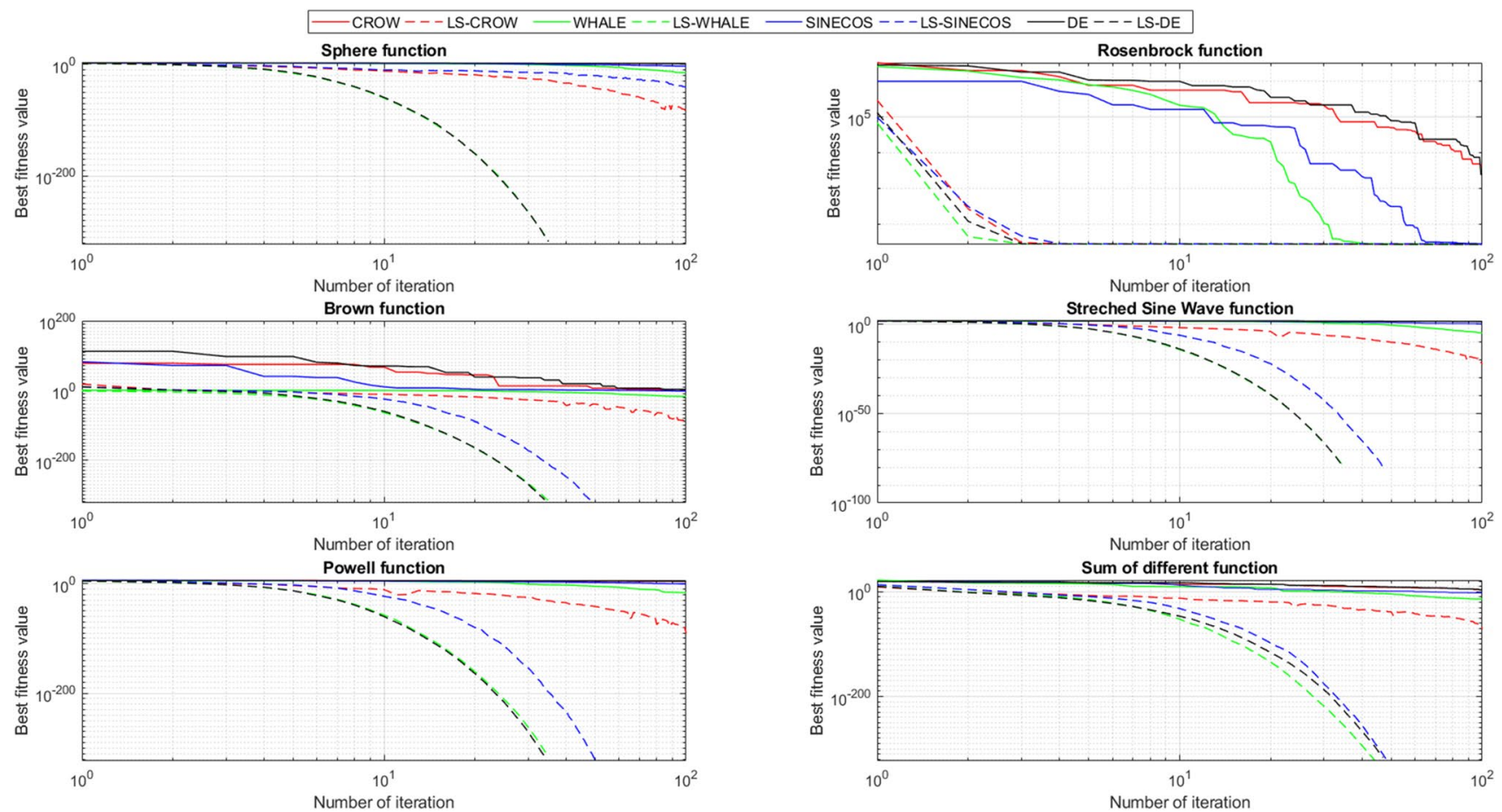

Fig. 8 Evolution characteristics of the compared algorithms for unimodal test functions from $f_{23}$-Sphere to $f_{28}-$ Sum of different functions
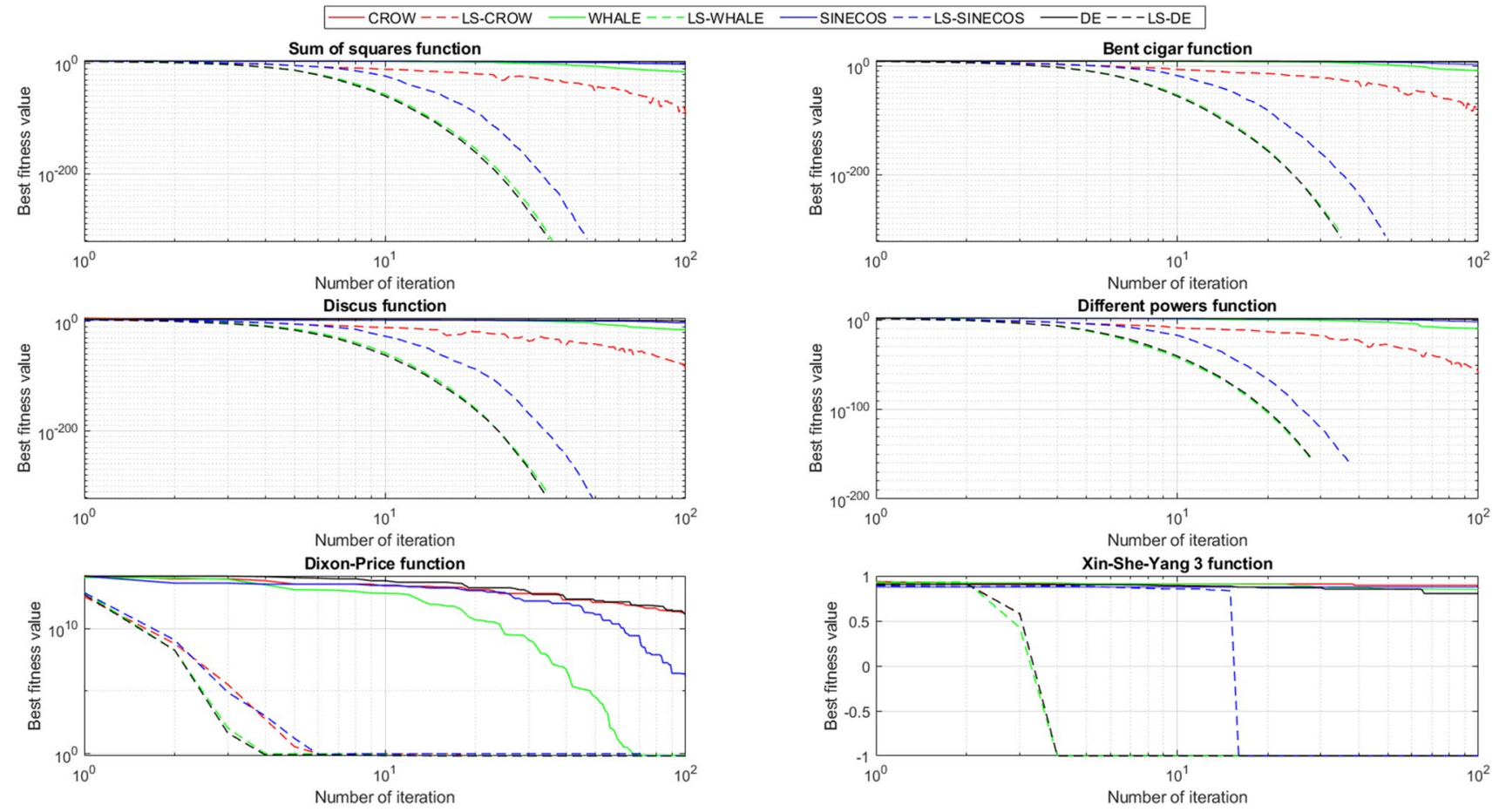

Fig. 9 Convergence analysis of the unimodal test functions from $f_{29}$-Sum of squares to $f_{34}$-Yang 


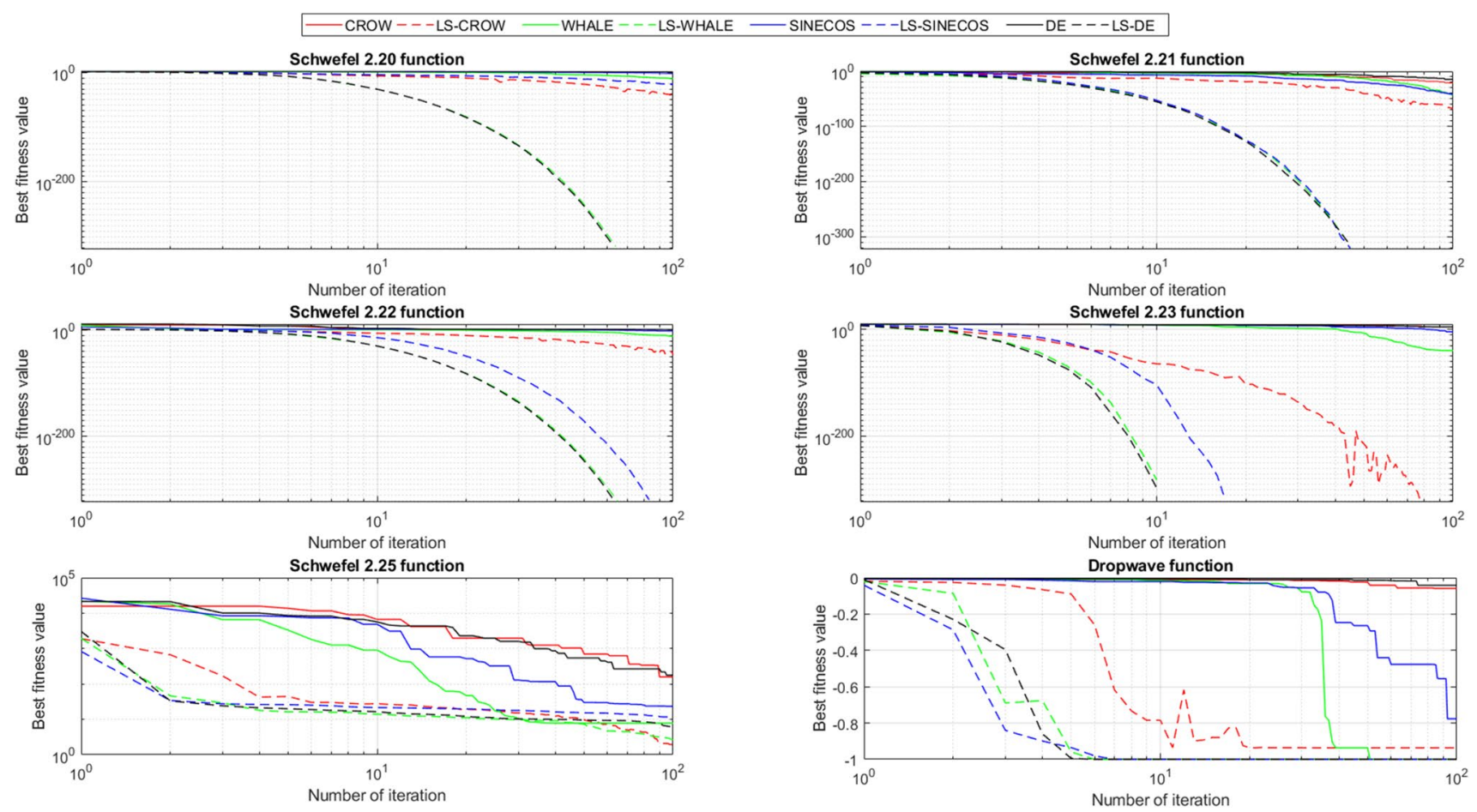

Fig. 10 Convergence characteristics of the compared algorithm for unimodal test functions from $f_{35}$-Schwefel 2.20 to $f_{40}$-Dropwave

$\arg \min (f(x))$

$$
\begin{aligned}
& f(x)=63098.88 x_{2} x_{4} x_{12}+5441.5 x_{2}^{2} x_{12}+115055 x_{2}^{1.664} x_{6} \\
& +6172.27 x_{2}^{2} x_{6}+63098.88 x_{1} x_{3} x_{11}+5441.5 x_{1}^{2} x_{11} \\
& +115055.5 x_{1}^{1.664} x_{5}+6172.27 x_{1}^{2} x_{5}+140.53 x_{1} x_{11} \\
& +281.29 x_{3} x_{11}+70.26 x_{1}^{2}+281.29 x_{1} x_{3}+281.20 x_{3}^{2} \\
& +14437 x_{1}^{2} x_{7} x_{8}^{1.8812} x_{9}^{-1} x_{10} x_{12}^{0.3434} x_{14}^{-1}+20470.2 x_{1}^{2} x_{7}^{2.893} x_{11}^{0.316}
\end{aligned}
$$

With subjected to below-defined constraints:
Table 6 reports the optimal results along with their corresponding constraint function values obtained for the refrigeration system design problem. The known best optimal solution for this problem is reported $\vec{x}=\{0.001,0.001,0.001,0.001,0.001,0.001,1.524,1.524$, $5.000,2.000,0.001,0.001,0.0072934,0.087558\} \quad f(\vec{x})=$ 0.32213 in Andrei [31]. It is seen that CL-DE slightly

$g_{1}(\vec{x})=1.524 x_{7}^{-1}-1.0 \leq 0, g_{2}(\vec{x})=1.524 x_{8}^{-1}-1.0 \leq 0$,

$g_{3}(\vec{x})=0.07789 x_{1}-2 x_{7}^{-1} x_{9}-1.0 \leq 0$,

$g_{4}(\vec{x})=7.05305 x_{1}^{2} x_{2}^{-1} x_{8}^{-1} x_{9}^{-1} x_{10} x_{14}^{-1}-1.0 \leq 0$,

$g_{5}(\vec{x})=0.0833 x_{13}^{-1} x_{14}-1.0 \leq 0$,

$g_{6}(\vec{x})=47.136 x_{2}^{0.33} x_{10}^{-1} x_{12}-1.333 x_{8} x_{13}^{2.1195}+62.08 x_{8}^{0.2} x_{10}^{-1} x_{12}^{-1} x_{13}^{2.1195}-1.0 \leq 0$,

$g_{7}(\vec{x})=0.0477 x_{8}^{1.8812} x_{10} x_{12}^{0.3424}-1.0 \leq 0$,

$g_{8}(\vec{x})=0.0488 x_{7}^{1.893} x_{9} x_{11}^{0.316}-1.0 \leq 0$,

$g_{9}(\vec{x})=0.0099 x_{1} x_{3}^{-1}-1.0 \leq 0$,

$g_{10}(\vec{x})=0.0193 x_{2} x_{4}^{-1}-1.0 \leq 0$,

$g_{11}(\vec{x})=0.0193 x_{2} x_{4}^{-1}-1.0 \leq 0$,

$g_{12}(\vec{x})=0.0298 x_{1} x_{5}^{-1}-1.0 \leq 0$,

$g_{13}(\vec{x})=0.056 x_{2} x_{6}^{-1}-1.0 \leq 0$,

$g_{14}(\vec{x})=2.0 x_{9}^{-1}-1.0 \leq 0$,

$g_{15}(\vec{x})=2.0 x_{10}^{-1}-1.0 \leq 0$,

$g_{16}(\vec{x})=x_{11}^{-1} x_{12}-1.0 \leq 0$ 
Table 6 Optimal results for the industrial refrigeration system design problem
Fig. 11 Schematic representation of the heat exchanger network design problem

\begin{tabular}{|c|c|c|c|c|c|c|}
\hline & CL-DE & $\mathrm{DE}$ & CL-WHALE & WHALE & CL-CROW & CL-SINECOS \\
\hline$x_{1}$ & 0.00100 & 0.00100 & 0.00100 & 0.00100 & 0.00100 & 0.00107 \\
\hline$x_{2}$ & 0.00100 & 0.00101 & 0.00100 & 0.00100 & 0.00100 & 0.00130 \\
\hline$x_{3}$ & 0.00100 & 0.00102 & 0.00100 & 0.00100 & 0.00100 & 0.00196 \\
\hline$x_{4}$ & 0.00100 & 0.00146 & 0.00100 & 0.00100 & 0.00100 & 0.00353 \\
\hline$x_{5}$ & 0.00100 & 0.00104 & 0.00100 & 0.00100 & 0.00100 & 0.00154 \\
\hline$x_{6}$ & 0.00100 & 0.00100 & 0.00100 & 0.00100 & 0.00100 & 0.00189 \\
\hline$x_{7}$ & 1.52413 & 1.52402 & 1.52400 & 1.52400 & 1.52400 & 1.73915 \\
\hline$x_{8}$ & 1.52404 & 1.52402 & 1.52400 & 1.52400 & 1.52400 & 1.58190 \\
\hline$x_{9}$ & 4.99999 & 4.99999 & 4.99999 & 4.99999 & 4.99842 & 4.60145 \\
\hline$x_{10}$ & 2.00000 & 2.00016 & 2.00000 & 2.32757 & 2.00558 & 2.74751 \\
\hline$x_{11}$ & 0.00100 & 0.00189 & 0.00100 & 0.01398 & 0.00159 & 0.00819 \\
\hline$x_{12}$ & 0.00100 & 0.00172 & 0.00100 & 0.01398 & 0.00157 & 0.00721 \\
\hline$x_{13}$ & 0.00727 & 0.00940 & 0.00729 & 0.02686 & 0.00896 & 0.01914 \\
\hline$x_{14}$ & 0.08729 & 0.11284 & 0.08760 & 0.32252 & 0.10762 & 0.21974 \\
\hline$g_{1}(x)$ & $-9.02 \mathrm{E}-5$ & $-1.46 \mathrm{E}-5$ & $-8.65 \mathrm{E}-8$ & $-6.8 \mathrm{E}-11$ & $-1.33 \mathrm{E}-7$ & -0.12371 \\
\hline$g_{2}(x)$ & $-3.07 E-5$ & $-4.28 \mathrm{E}-5$ & $-6.87 \mathrm{E}-7$ & $-3.1 \mathrm{E}-10$ & $-7.75 \mathrm{E}-5$ & -0.03660 \\
\hline$g_{3}(x)$ & -7.56100 & -7.56149 & -7.56159 & -7.56160 & -7.55952 & -6.29152 \\
\hline$g_{4}(x)$ & -0.97879 & -0.98385 & -0.97886 & -0.99332 & -0.98276 & -0.98939 \\
\hline$g_{5}(x)$ & -5.01206 & $-3.01 E-4$ & $-3.20 \mathrm{E}-6$ & $-4.7 \mathrm{E}-10$ & $-2.94 \mathrm{E}-6$ & -0.04394 \\
\hline$g_{6}(x)$ & -0.00619 & -0.00616 & $-8.61 \mathrm{E}-7$ & $-2.51 \mathrm{E}-9$ & -0.01596 & -0.20187 \\
\hline$g_{7}(x)$ & -0.98019 & -0.97612 & -0.98019 & -0.94313 & -0.97066 & -0.94261 \\
\hline$g_{8}(x)$ & -0.93892 & -0.92525 & -0.93891 & -0.85945 & -0.92922 & -0.85975 \\
\hline$g_{9}(x)$ & -0.99009 & -0.99035 & -0.99010 & -0.99010 & -0.99009 & -0.99461 \\
\hline$g_{10}(x)$ & -0.98070 & -0.98658 & -0.98071 & -0.98070 & -0.98106 & -0.99285 \\
\hline$g_{11}(x)$ & -0.97019 & -0.97160 & -0.97020 & -0.97020 & -0.97066 & -0.97936 \\
\hline$g_{12}(x)$ & -0.94400 & -0.94318 & -0.94399 & -0.94400 & -0.94616 & -0.96122 \\
\hline$g_{13}(x)$ & -0.59999 & -0.59999 & -0.59999 & -0.59999 & -0.59987 & -0.56535 \\
\hline$g_{14}(x)$ & $-1.27 \mathrm{E}-5$ & $-8.42 \mathrm{E}-5$ & $-1.71 \mathrm{E}-6$ & -0.14073 & -0.00207 & -0.27206 \\
\hline$g_{15}(x)$ & $-3.17 \mathrm{E}-5$ & -0.08907 & $-6.73 E-5$ & $-5.60 \mathrm{E}-9$ & -0.01557 & -0.11971 \\
\hline Min & 0.03228 & 0.03329 & 0.03229 & 0.04503 & 0.03288 & 0.07607 \\
\hline Mean & 0.04818 & 0.03656 & 0.03240 & 0.05465 & 0.04751 & 0.10001 \\
\hline SD & 0.00885 & 0.00323 & $1.18 \mathrm{E}-4$ & 0.00707 & 0.01066 & 0.01849 \\
\hline Worst & 0.07443 & 0.04694 & 0.03262 & 0.07201 & 0.07365 & 0.15515 \\
\hline
\end{tabular}

Bold values are the best solutions obtained by the corresponding optimization algorithm for the related problem

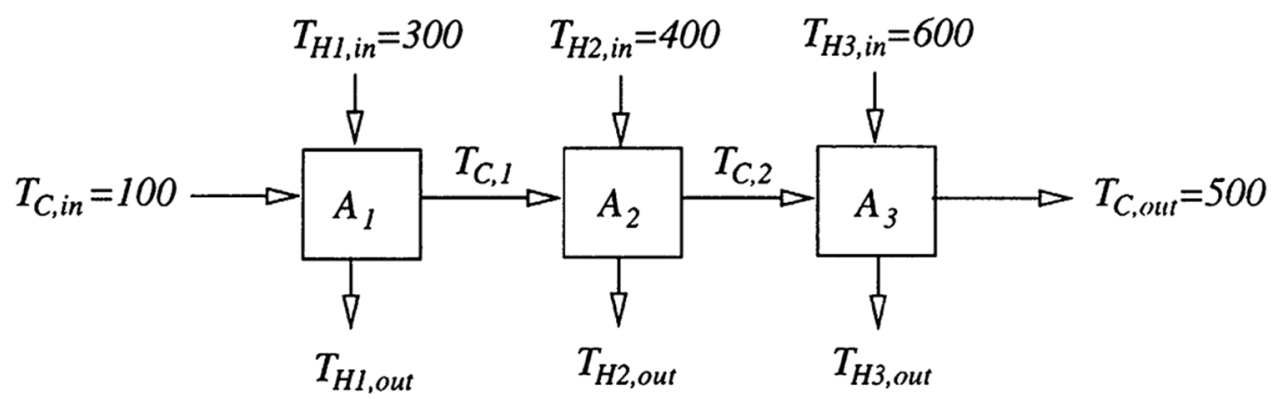

outperforms standard DE and in terms of minimum objective function value of 0.03228 , however, the solution robustness of $\mathrm{DE}$ is better than $\mathrm{CL}-\mathrm{DE}$. Improvement made by the local search method in the quality of solutions for the WHALE algorithm is more significant compared to that of the DE method. Besides, it is interesting to see that 
Table 7 Optimal results for the heat exchanger network design problem

\begin{tabular}{|c|c|c|c|c|c|c|c|c|}
\hline & CL-DE & $\mathrm{DE}$ & CL-CROW & CROW & CL-WHALE & WHALE & CL-SINECOS & SINECOS \\
\hline$x_{1}$ & 1073.9493 & 138.8717 & 1050.4774 & 1045.6817 & 104.6838 & 908.4932 & 781.3191 & 593.4413 \\
\hline$x_{2}$ & 1000.0000 & 3933.3608 & 1000.0039 & 1000.0670 & 1000.0002 & 1000.0042 & 1035.0089 & 1302.346 \\
\hline$x_{3}$ & 5439.4390 & 6597.0548 & 5571.2750 & 6279.5585 & 7443.4502 & 7628.1219 & 6437.4597 & 6634.997 \\
\hline$x_{4}$ & 268.3603 & 99.5445 & 264.5470 & 255.1644 & 130.6153 & 231.8829 & 240.2524 & 210.764 \\
\hline$x_{5}$ & 282.4224 & 240.9296 & 277.3403 & 250.1843 & 202.3045 & 195.1698 & 265.3606 & 252.179 \\
\hline$x_{6}$ & 131.6396 & 122.9564 & 131.5335 & 124.6549 & 252.2818 & 125.4751 & 151.5722 & 174.504 \\
\hline$x_{7}$ & 285.9379 & 146.4110 & 281.7915 & 302.7145 & 224.4625 & 260.3007 & 274.7753 & 254.503 \\
\hline$x_{8}$ & 382.4224 & 339.2508 & 377.2544 & 349.8019 & 302.2948 & 295.0732 & 361.0858 & 345.699 \\
\hline$g_{1}(x)$ & 0.0000 & -177.4990 & -3.91941 & -20.1806 & -17.1027 & -42.6418 & -8.1752 & -14.7310 \\
\hline$g_{2}(x)$ & 0.0000 & -12.2038 & -5.41509 & -2.2656 & -3.8481 & -76.4124 & -0.1165 & -4.0817 \\
\hline$g_{3}(x)$ & 0.0000 & -1.6787 & -0.08587 & -0.3823 & -0.0097 & -0.09658 & -4.2747 & -6.4802 \\
\hline$g_{4}(x)$ & 0.0000 & -17315.88 & $-8.17 \mathrm{E}-9$ & $-4.0 \mathrm{E}-6$ & -792.3699 & -3182.384 & -767.9452 & -10660.5 \\
\hline$g_{5}(x)$ & 0.0000 & -7611.516 & -1252.93 & -53778.49 & -4235.717 & -74309.32 & -4346.223 & -5193.93 \\
\hline$g_{6}(x)$ & 0.0000 & -954.7732 & $-4.43 E-5$ & -1015.61 & -33.713 & $-4.270 \mathrm{E}-4$ & -29628.80 & -952.632 \\
\hline Min & 7513.3883 & $10,669.287$ & 7621.7565 & 8325.307 & 8548.1344 & 9536.619 & 8253.7878 & 8530.785 \\
\hline Mean & 329.2731 & 2637.601 & 614.2513 & 2470.965 & 289.6435 & 1398.656 & 286.3433 & 361.496 \\
\hline SD & 7933.2837 & $14,689.216$ & 8653.1931 & $12,239.723$ & 9052.0461 & $11,650.818$ & 8770.0161 & 9428.840 \\
\hline Worst & 9803.6892 & $21,104.501$ & $10,001.951$ & $18,471.314$ & 9781.1586 & $14,480.662$ & 9141.7638 & $10,256.548$ \\
\hline
\end{tabular}

Bold values are the best solutions obtained by the corresponding optimization algorithm for the related problem

standard CROW and SINECOS optimizers fail to find a feasible solution in any algorithm run, therefore these methods do not take place in Table 6. However, CL-CROW is the second-best performing optimizer after CL-DE. Among the compared methods, CL-SINECOS provides the worst predictive performance.

\subsection{Optimal design of a heat exchanger network}

A cold stream at $100 \mathrm{~F}$ is subjected to be heated to 500 $F$ employing three hot streams having different inlet temperatures as illustrated in Fig. 11. Relying on the fact that temperatures of hot streams along with their corresponding thermophysical characteristics flowing through the series of heat exchangers are known and given in the below set of equations, it is aimed to obtain the optimal outlet temperatures of each stream which provides the minimum total heat exchange surface. That is, the optimization goal, in this case, is to find the set of optimal design variables resulting in a minimum total area of heat exchangers [31]. The mathematical formulation of this design problem can be expressed by the following set of equations

$\arg \min f(\vec{x}) \quad \vec{x}=\left\{A_{1}, A_{2}, A_{3}, T_{C 1}, T_{C 2}, T_{H 1, \text { out }}, T_{H 2, \text { out }}, T_{H 3, \text { out }}\right\}$ $f(\vec{x})=A_{1}+A_{2}+A_{3}$

Subjected to below-given design constraints
$g_{1}(\vec{x})=T_{C 1}+T_{H 1, \text { out }}-T_{C, \text { in }}-T_{H 1, \text { in }} \leq 0$

$g_{2}(\vec{x})=-T_{C 1}+T_{C 2}+T_{H 2, \text { out }}-T_{H 1, \text { in }} \leq 0$

$g_{3}(\vec{x})=T_{H 3, \text { out }}-T_{C 2}-T_{H 3, \text { in }}+T_{C, \text { out }} \leq 0$

$g_{4}(\vec{x})=A_{1}-A_{1} T_{H 1, \text { out }}+\frac{F C_{p}}{U_{1}} T_{C 1}-\frac{F C_{p}}{U_{1}} T_{C, \text { in }} \leq 0$

$g_{5}(\vec{x})=A_{2} T_{C 1}-A_{2} T_{H 2, \text { out }}-\frac{F C_{p}}{U_{2}} T_{C 1}+\frac{F C_{p}}{U_{2}} T_{C 2} \leq 0$

$g_{6}(\vec{x})=A_{3} T_{C 2}-A_{3} T_{H 3, \text { out }}-\frac{F C_{p}}{U_{3}} T_{C 2}+\frac{F C_{p}}{U_{3}} T_{C, \text { out }} \leq 0$

$100 \leq A_{1} \leq 10,000,1000 \leq A_{2}, A_{3} \leq 10,000$,

$10 \leq T_{C 1} \leq 1000,10 \leq T_{C 2} \leq 1000,10 \leq T_{H 1, \text { out }} \leq 1000$,

$10 \leq T_{H 2, \text { out }} \leq 1000,10 \leq T_{H 3, \text { out }} \leq 1000$,

$F C_{p}=10^{5}$ and $U=[120,80,40]$.

where $A_{1}, A_{2}$, and $A_{3}$ are the respective heat transfer areas of the first, second, and third heat exchangers lined up in series. Outlet temperatures of the cold streams circulating through the first and second heat exchangers are respectively symbolized by $T_{C 1}$ and $T_{C 2}$. Outlet temperatures of the hot streams are respectively represented by the symbols of $T_{\mathrm{H} 1 \text {,out }}, T_{\mathrm{H} 2 \text {,out }}$ and $T_{\mathrm{H} 3 \text {,out }}$. It is also worth mentioning that inlet and outlet stream temperatures are defined in the Fahrenheit scale. The best optimum known solution for this problem is provided in Andrei [31] given as $\vec{x}=\{1026.948,1000.0,5485.282,265.060,280.589$, 
$134.940,284.471,380.589\}$ at $f(\vec{x})=7512.230$. Table 7 reports the optimal results by the compared metaheuristic algorithms. CL-DE obtains the minimum objective function value $f(\vec{x})=7513.3883$ without violating any imposed design constraints. CL-CROW becomes the second-best performing optimizer with the corresponding fitness function value of $f(\vec{x})=7621.7565$. It is also seen that using the proposed hybrid local search scheme as a performance booster for the mentioned metaheuristic algorithms works well for this optimization problem, which is deduced by the improved solution quality and robustness maintained by the local search procedure.

\section{Solving transient heat conduction problems}

In this section, a one-dimensional transient heat conduction problem will be solved by the abovementioned metaheuristic algorithms along with their local search boosted versions. Temperature distribution as a function of time and space $T(x, t)$ in the medium is conventionally computed by applying various types of root-finding methods. In the context of this research study, root finding equation that represents the solution of the problem is converted into an optimization problem and solved by the above-mentioned optimization algorithms, which can be conceptualized as an alternative solution strategy eliminating the tedious and time-consuming location of good initial estimates within the respective root-finding region. Any erroneous or deceptive estimation of these first values leads to unexpected solution outcomes that may be far away from the exact roots of the problem. Utilizing optimization algorithms in this concept automates detecting the candidate roots within the defined search region which are, in essence, optimal solutions of the transformed optimization problem.

In the most general form, the three-dimensional heat diffusion in a medium is expressed by the following equation defined in Cartesian coordinates

$\frac{\partial}{\partial x}\left(k \frac{\partial T}{\partial x}\right)+\frac{\partial}{\partial y}\left(k \frac{\partial T}{\partial y}\right)+\frac{\partial}{\partial z}\left(k \frac{\partial T}{\partial z}\right)+\dot{q}=\rho C_{p} \frac{\partial T}{\partial t}$

where $k$ is the thermal conductivity; $\dot{q}$ is the rate of heat generated in the medium; $\rho$ and $C_{p}$ are respectively density and specific heat of the medium in which the available heat is diffused. This equation is referred to the heat equation and provides a basic application to obtain a spatial temperature distribution $T(x, y, z)$ as a function of time $(t)$ in any kind of heat exchange medium. However, some modifications can be made on Eq. (7) to eliminate unnecessary quantities. Suppose that heat transfer is one-dimensional and no heat generation occurs within the medium. Then, the modified heat equation without taking into account these terms becomes

$\frac{\partial^{2} T}{\partial x^{2}}=\frac{1}{\alpha} \frac{\partial T}{\partial t}$

where $\alpha=\frac{k}{\rho c_{p}}$ is the thermal diffusivity. This differential equation can be solved if the initial conditions and boundary conditions of the heat transfer medium is specified. The initial conditions of the transient heat conduction problem can be expressed by

$T(x, 0)=T_{i}$

and the corresponding boundary conditions are

$\left.\frac{\partial T}{\partial x}\right|_{x=0}=0$

and

$-\left.k \frac{\partial T}{\partial x}\right|_{x=L}=h\left[T(L, t)-T_{\infty}\right]$

Where $h$ is the convective heat transfer coefficient, $L$ is the length of the medium and $T_{\infty}$ is the ambient temperature. Equation (9) explains the uniform temperature distribution along with the medium at initial time $t=0$. Equation (10) reflects the zero heat flux to the medium at $x=0$ and Eq. (11) describes the rate of heat convection at $x=L$ when $t>0$. From Eq. (9) to Eq. (11), one can easily comprehend that one-dimensional temperature distribution across the medium heavily depends on time $(t)$ and space $(x)$ along with the functional physical parameters given below

$T=T\left(x, t, k, h, \alpha, L, T_{i}, T_{\infty}\right)$

This problem can be either solved analytically or numerically. Non-dimensionalizing the governing equations eases the path of solution steps of these types of problems. For instance, take the dependent variable temperature $T$ as a function of time and space. The temperature difference $\phi=T-T_{\infty}$ is divided by the maximum temperature difference $\phi_{i}=T_{i}-T_{\infty}$ to obtain a dimensionless dependent temperature variable which is equated by

$\phi^{*}=\frac{\phi}{\phi_{i}}=\frac{T-T_{\infty}}{T_{i}-T_{\infty}}$

And the dimensionless form of the spatial coordinate can be alternatively expressed by the following

$x^{*}=\frac{X}{L}$

where $L$ is the half-thickness of the heat transfer medium. The dimensionless form of the elapsed time can be 
Fig. 12 Schematic view of a infinite wall and $\mathbf{b}$ sphere as a heat transfer medium

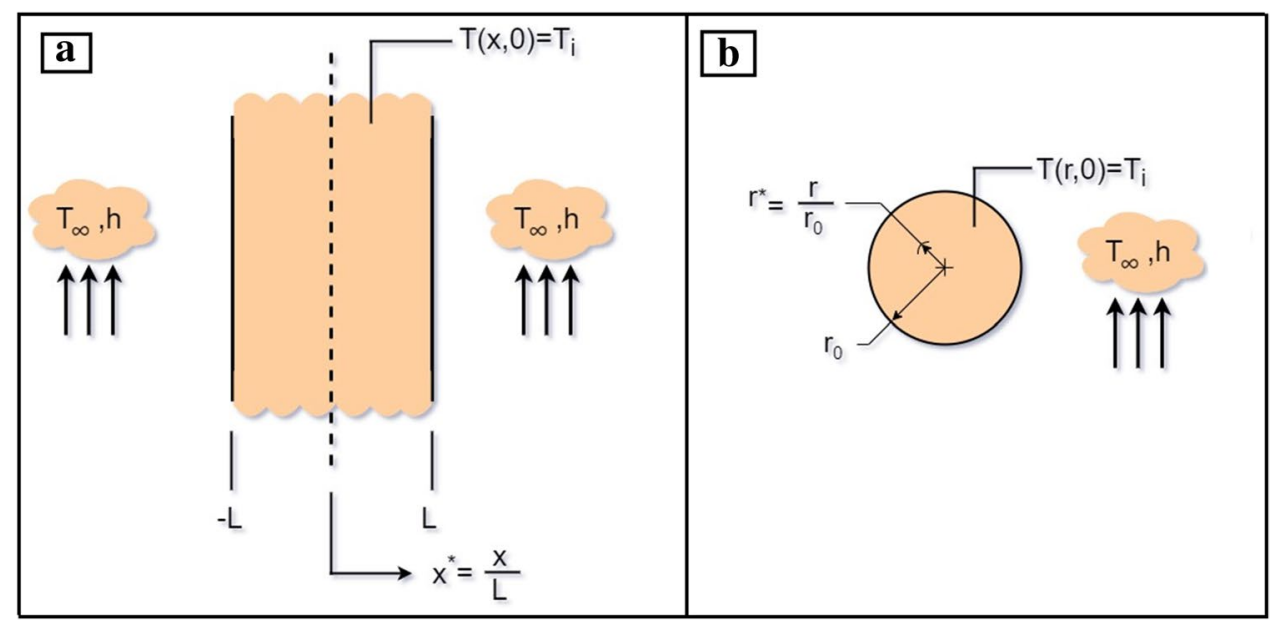

reformed in terms of Fourier number and can be formulated by

Fo $=\frac{\alpha \cdot t}{L^{2}}=t^{*}$

where $\alpha$ is the thermal diffusivity in $\mathrm{m}^{2} / \mathrm{s}$ and $t$ is the elapsed time in seconds. Equation (9) to Eq. (11) can be reformulated using dimensionless numbers defined in Eq. (13) to Eq. (15). Thereby, the one-dimensional heat dissipation equation becomes

$\frac{\partial^{2} \phi^{*}}{\partial x^{* 2}}=\frac{\partial \phi^{*}}{\partial F o}$

and the corresponding initial and boundary conditions take the final form of

$\phi^{*}\left(x^{*}, 0\right)=1$

$\left.\frac{\partial \phi^{*}}{\partial x^{*}}\right|_{x^{*}=0}=0$

$\left.\frac{\partial \phi^{*}}{\partial x^{*}}\right|_{x^{*}=1}=-B i \cdot \phi^{*}\left(1, t^{*}\right)$

where $B i=h \cdot L / k$ is the dimensionless Biot number. The functional dependence of Eq. (12) can be re-expressed in nondimensionalized form by the following equation

$\phi^{*}=f\left(x^{*}, F O, B i\right)$

A significant advantage is gained by casting the problem into a dimensionless form as reformulated in Eq. (20). With such reformulation, universal representation of temperature distribution in any type of geometry in dimensionless form only depends on $x^{*}$, Fo, and Bi. Therefore, the dimensionless solution will not be dependent on a particular value of $h, \alpha, k, L, T_{\infty}, T_{i}$. Nondimensionalization in that form will greatly simplify the analytic solution procedure prescribed for solving transient heat conduction problems.

Many types of analytical solution techniques have been proposed for solving transient heat conduction problems for different simplified geometries having various kinds of boundary conditions. One of the most common mathematical models is the separation of variables method which is a convenient solution strategy to attain dimensionless temperature distribution across the heat transfer medium in the mathematical form of infinite series. Suppose that one-dimensional temperature distribution is expected to be obtained for a plane wall of thickness $2 L$ as depicted in Fig. 12a. If the thickness of the wall is negligible to its respective length, it is reasonable to presume that heat conduction occurs only in $x$-direction. Assuming that the uniform initial temperature of the wall at $t=0$ is $T_{i}$, that is $T(x, 0)=T_{i}$ and the wall is suddenly immersed in a heat transfer environment at a uniform temperature of $T_{\infty}$ where $T_{i} \neq T_{\infty}$ corresponding temperature distribution across the one-dimensional wall can be obtained by solving the differential equation defined in Eq. (16) subjected to the series of boundary conditions in the dimensionless form as given in Eq. (17) to Eq. (19). Since the boundary conditions for two symmetric surfaces $x^{*}= \pm 1$ are the same, temperature distribution along the $x$-direction at any time instant should be symmetric to the midplane $\left(x^{*}=0\right)$. Based on the imposed boundary conditions, the exact analytic solution to this problem can be expressed as [32]

$\phi^{*}=\sum_{n=1}^{\infty} A_{n} \exp \left(-\beta_{n}^{2} F o\right) \cos \left(\beta_{n} X^{*}\right)$

Where Fo is the Fourier number expressed in Eq. (15) and $A_{n}$ the coefficient is defined as a function of $\beta_{n}$ by the below given transcendental equation 


$$
A_{n}=\frac{4 \sin \left(\beta_{n}\right)}{2 \beta_{n}+\sin \left(2 \beta_{n}\right)}
$$

Eigenvalues $\beta_{n}$ of Eq. (22) are the positive roots of the following transcendental equation

$\beta_{n} \tan \left(\beta_{n}\right)=B i$
Where $B i$ is the Biot number defined in the above paragraphs. From Eq. (21) to Eq. (23), it can be concluded that once the eigenvalues $\beta_{n}$ are calculated from the transcendental equation Eq. (23), series of $A_{n}$ coefficients become known quantities and dimensionless temperature distribution $\phi^{*}$ will be easily obtained by Eq. (21). However, problems may arise in retaining accurate solutions of the transcendental equation, which necessitates the application of the appropriate algorithmic procedure. There are several

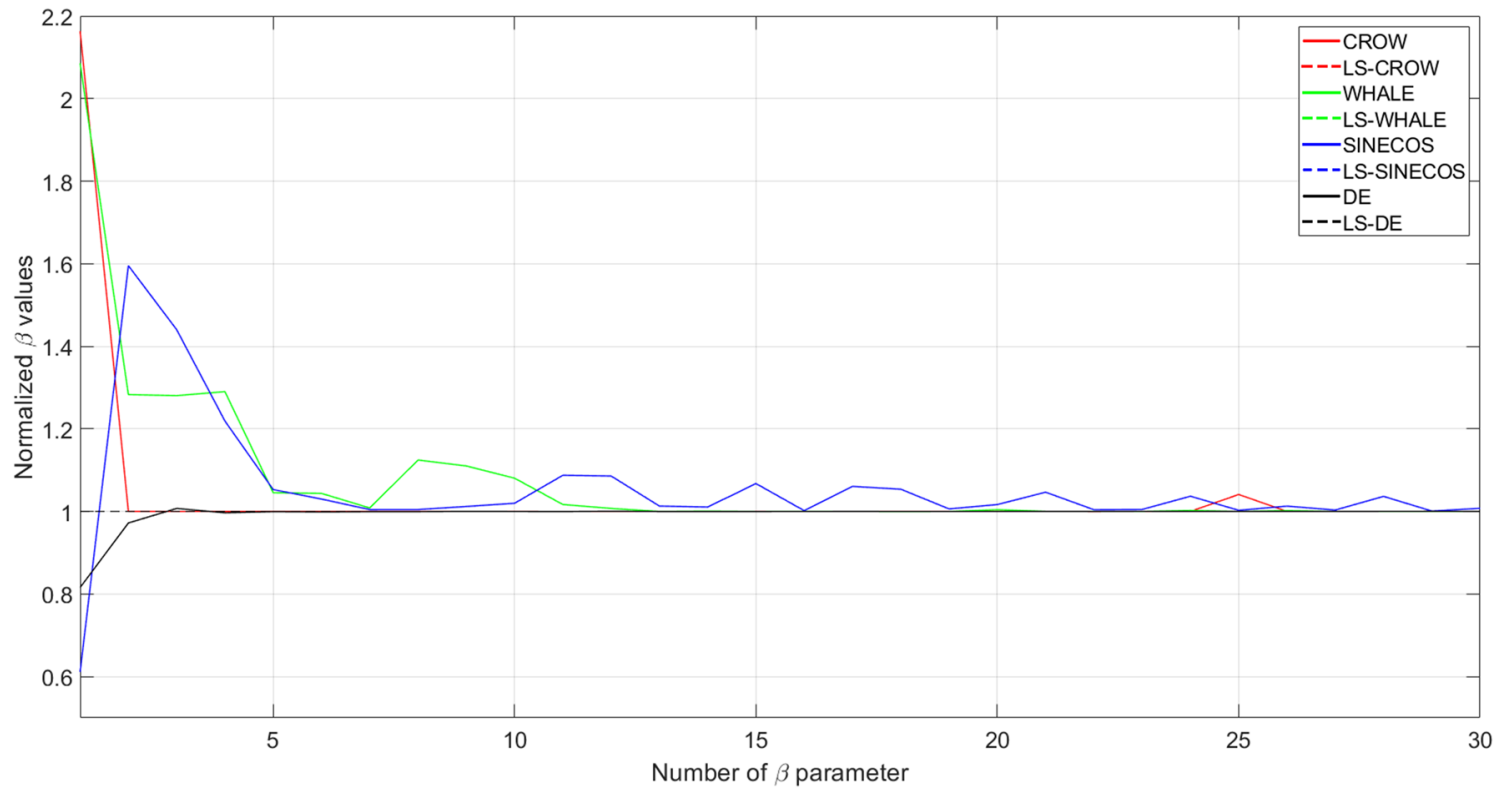

Fig. 13 Normalized $\beta$ eigenvalues obtained by different optimization algorithms

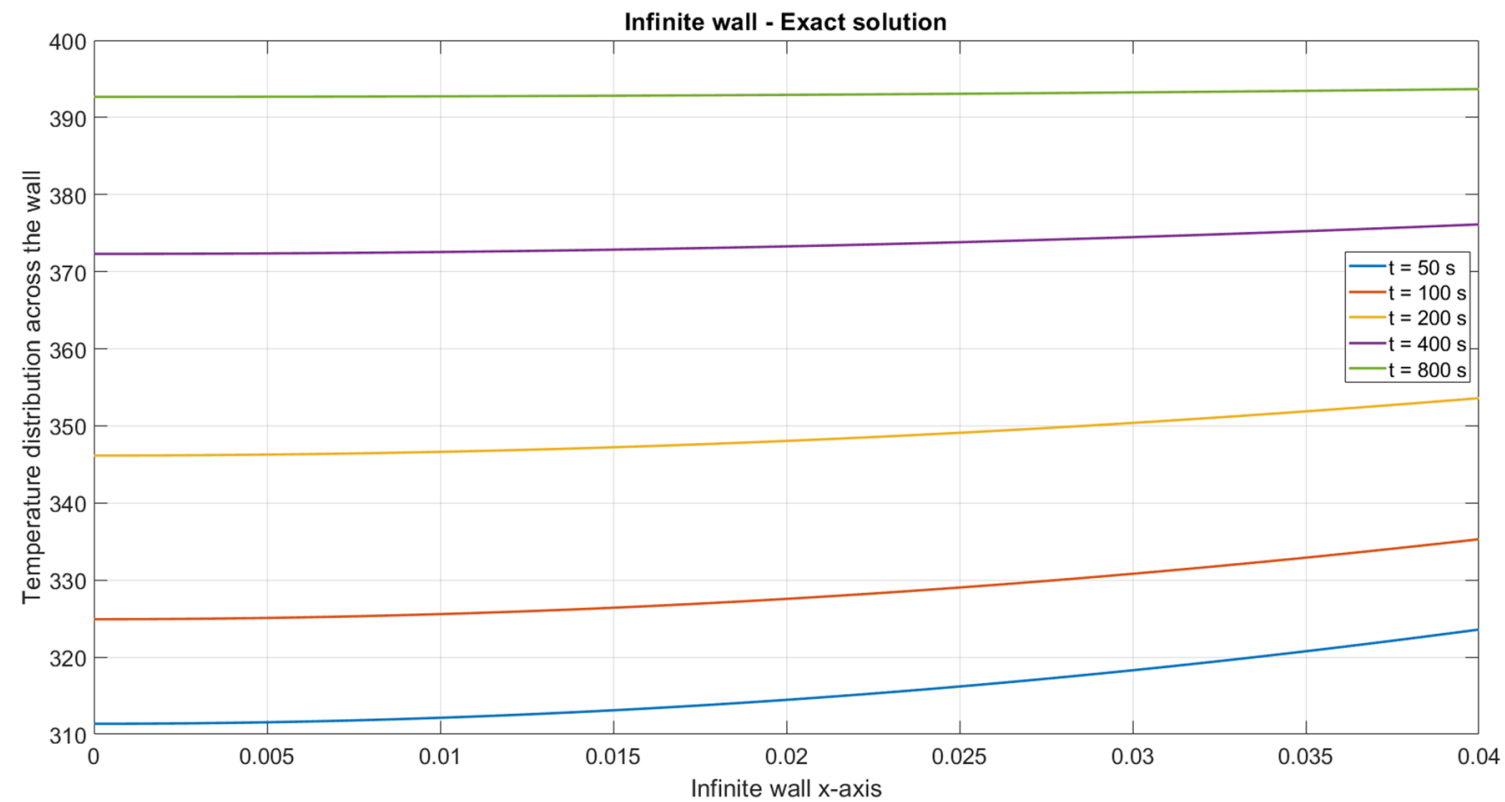

Fig. 14 Temperature distribution across the infinite wall obtained by the Secant root finding method 


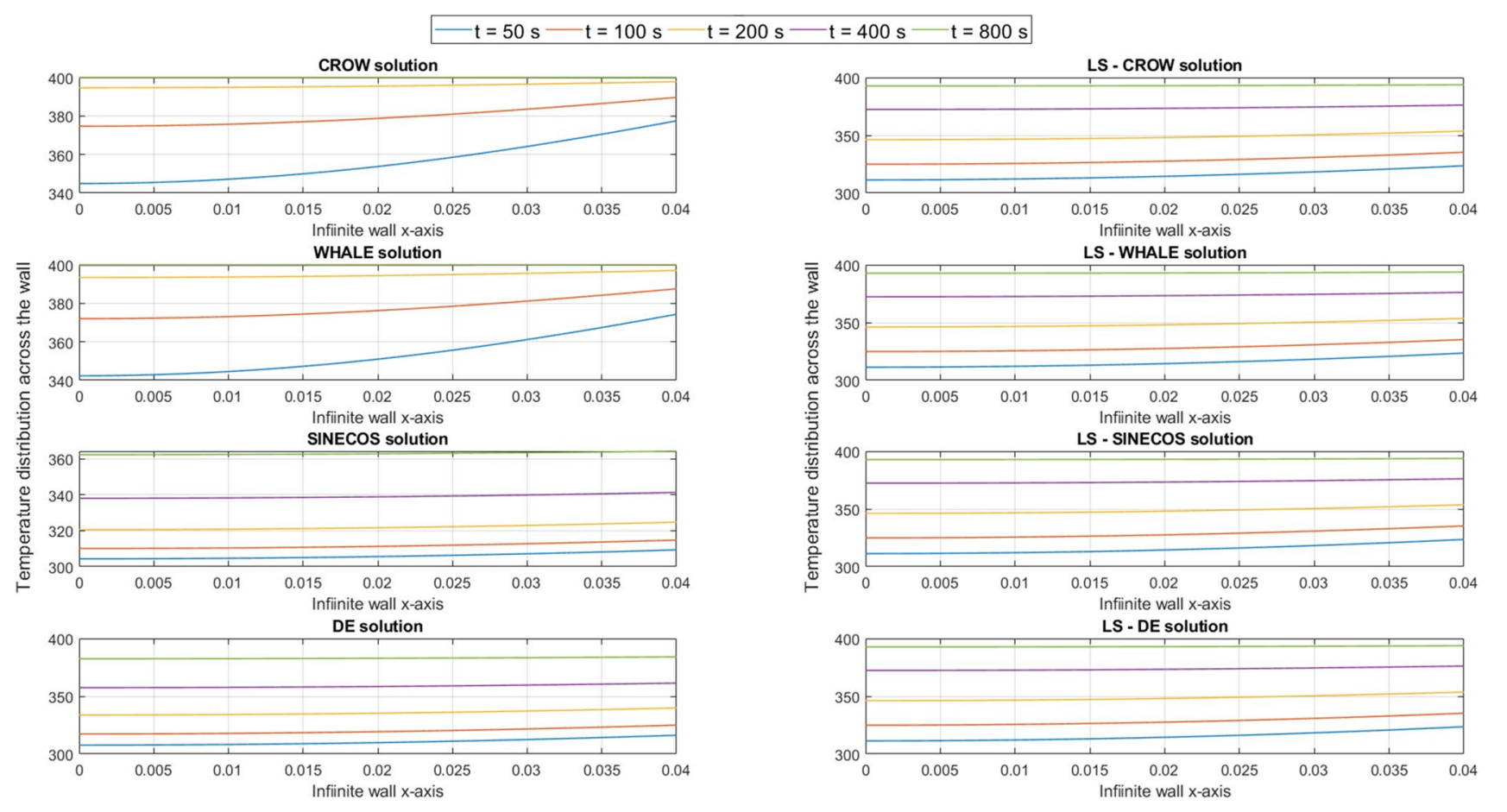

Fig. 15 Estimated temperature distribution through the compared metaheuristic algorithms

Table 8 Statistical results in terms of RMSE values to obtain temperature distribution across the infinite wall

\begin{tabular}{lllll}
\hline & Best & SD & Mean & Worst \\
\hline CROW & $7.10 \mathrm{E}+00$ & $4.97 \mathrm{E}+01$ & $5.48 \mathrm{E}+01$ & $2.10 \mathrm{E}+02$ \\
CL-CROW & $\mathbf{3 . 5 2 E}-06$ & $2.23 \mathrm{E}-01$ & $7.24 \mathrm{E}-02$ & $1.93 \mathrm{E}+00$ \\
WHALE & $1.98 \mathrm{E}+02$ & $9.35 \mathrm{E}+01$ & $3.72 \mathrm{E}+02$ & $5.27 \mathrm{E}+02$ \\
CL-WHALE & $1.18 \mathrm{E}-03$ & $2.46 \mathrm{E}-01$ & $9.00 \mathrm{E}-02$ & $1.30 \mathrm{E}+00$ \\
SINECOS & $6.38 \mathrm{E}+02$ & $6.13 \mathrm{E}+01$ & $7.95 \mathrm{E}+02$ & $9.02 \mathrm{E}+02$ \\
CL-SINECOS & $4.34 \mathrm{E}-03$ & $4.63 \mathrm{E}-01$ & $2.78 \mathrm{E}-01$ & $1.72 \mathrm{E}+00$ \\
DE & $1.01 \mathrm{E}+01$ & $2.01 \mathrm{E}+01$ & $3.29 \mathrm{E}+01$ & $5.64 \mathrm{E}+01$ \\
CL-DE & $7.39 \mathrm{E}-06$ & $2.31 \mathrm{E}-01$ & $9.03 \mathrm{E}-02$ & $1.30 \mathrm{E}+00$ \\
\hline
\end{tabular}

Bold values are the best solutions obtained by the corresponding optimization algorithm for the related problem

solution strategies available in the literature for solving Eq. (23) including graphical methods and different types of root-finding algorithms. Albeit, each different method has intrinsic drawbacks, complicating to obtain the accurate solution of the problem. Roots of the transcendental equations can be located by the graphical methods, however, it may not be feasible to successfully obtain the accurate position of each root utilizing graphical representation. Correct values of the roots of transcendental equations can be alternatively computed by root-finding algorithms such as the Bisection method, a derivativebased method of Newton-Raphson, Secant methods, etc.
However, these analytical root-finding methods require the correct location of the region where the related root lies. This can be possible in either of two ways. One option is to utilize graphical methods to locate the region of the root. Another option is to make good and plausible initial estimates for the iterative algorithmic procedure. Making promising estimates for each transcendental root may not be realized as it is some kind of a black-box process. To eliminate these difficulties in finding the correct numerical values of the transcendental roots, associated eigenvalues can be obtained by an optimization algorithm rather than a root-finding method. Equation (23) can be reformulated into an optimization problem with the below-defined error function

$f\left(\beta_{n}\right)=\beta_{n} \tan \left(\beta_{n}\right)-B i$

The fitness function of the optimization problem is formulated in terms of root mean square error (RMSE)

as expressed in the below-defined equation

$\arg \min f_{R M S E}\left(\beta_{n}\right)=\sqrt{\frac{1}{N} \sum_{n=1}^{N} f\left(\beta_{n}\right)^{2}}$

Where $N$ is the number of transcendental roots for solving Eq. (25), which also represents the dimensionality of the optimization problem and is considered as 30 for this study. Now consider an infinite wall whose initial uniform 


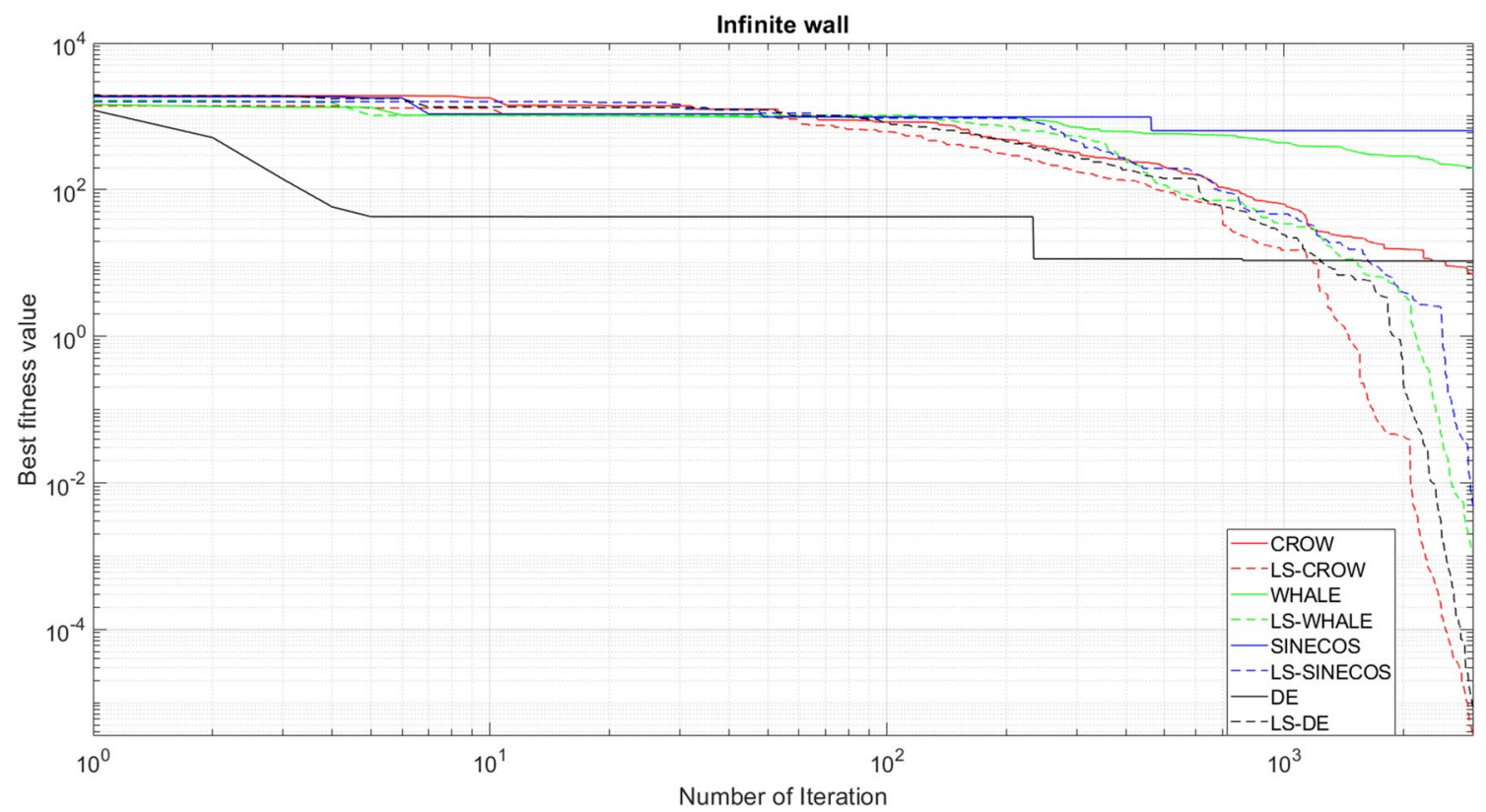

Fig. 16 Evolution behaviors of the compared algorithms for infinite wall problem

temperature is $T_{i}=300 \mathrm{Kelvin}(\mathrm{K})$, length in $x$-direction is $L=0.04 \mathrm{~m}$; thermal conductivity is $k=63.9 \mathrm{~W} / \mathrm{m}^{2} \mathrm{~K}$, and thermal diffusivity is $\alpha=18.8 \mathrm{E}-6 \mathrm{~m}^{2} / \mathrm{s}$. Sudden heat exchange occurs at $\mathrm{t}=0$ between the infinite wall and ambient whose convective heat transfer coefficient $h=200 \mathrm{~W} / \mathrm{m}^{2} \mathrm{~K}$ at $T_{\infty}=400 \mathrm{~K}$. Time-dependent temperature distribution along the one-dimensional infinite wall based on the above defined thermophysical properties can be obtained by using Eq. (18) to Eq. (20). Figure 13 visualizes the sequence of $\beta_{n}$ eigenvalues obtained by the metaheuristic algorithms whose corresponding numerical values are normalized with exact solutions obtained by the Secant root finding method. It is seen that deviations are higher at the initial steps then error rates are decreased to some extent for CROW, DE, WHALE, and SINECOS algorithms. Set of eigenvalues $\beta_{n}$ found by the local search improved algorithms thoroughly agree well with the exact solutions. Figure 14 depicts the exact temperature distribution across the infinite wall for different elapsed times retained by the Secant root finding method. Figure 15 compares the accuracy of temperature distribution values obtained by the metaheuristic algorithms and their local search enhanced versions. It is observed that local search embedded metaheuristics have the capability to predict the temperature distribution in the wall much more accurately than those estimated by the remaining algorithms shown in Fig. 15. Table 8 reports the deviation results for the infinite wall problem. CL-CROW performs the best predictive performance with the minimum objective function value of $3.52 \mathrm{E}-6$ among the compared algorithms followed by the $C L-D E$ algorithm having the best result of $7.39 \mathrm{E}-06$ obtained after competitive algorithm runs. The worst estimations between the local search enhanced methods are performed by CL-SINECOS with the best solution of 4.34E-03. Base algorithms of CROW, WHALE, SINECOS, and DE methods are not capable to retain any feasible results, even in a single algorithm run. However, CROW makes the best estimations between them with a minimum objective function value of $7.10 \mathrm{E}+00$. Figure 16 illustrates the convergence histories of the compared algorithms for this case. After a settled stagnation period within the early phases of iterations, the rapid and quick decline is observed on convergence rates for local search improved algorithms. This tendency in convergence behavior of the improved algorithms also proves the maintained balance between the exploration and exploitation as mentioned in the algorithm development section.

Now think of a sphere as a heat transfer medium with a radius length $r_{o}$ as depicted in Fig. 12b. This sphere at uniform initial temperature experiences a convective heat exchange between the surrounding ambient whose corresponding mathematical model describing the heat transfer mechanism is similar to that is developed for the infinite wall. Infinite series solution of one-dimensional radial temperature distribution along the sphere can be expressed in a nondimensionalized temperature difference form by the below-given equation [32] 


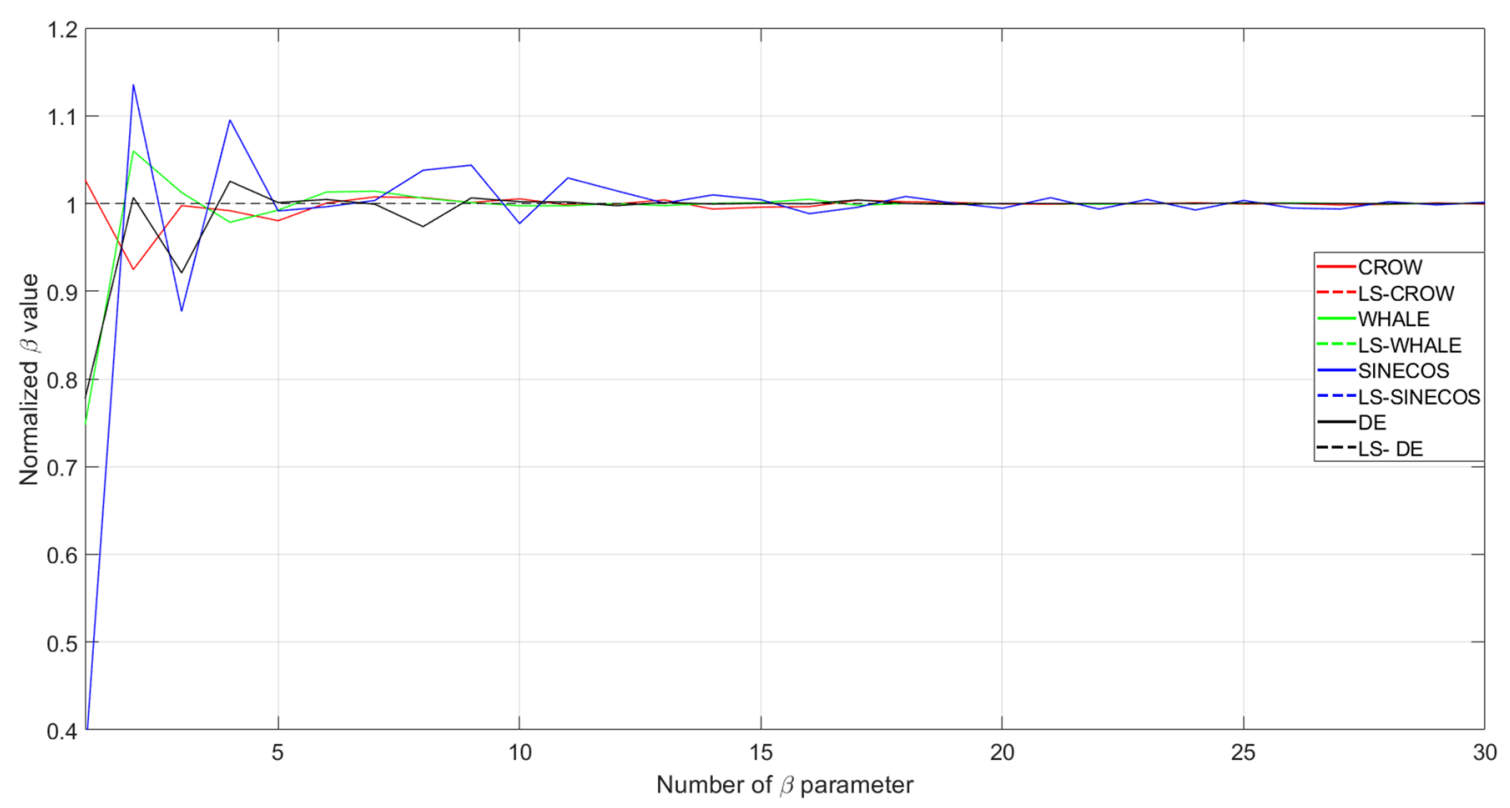

Fig. 17 Normalized $\beta$ eigenvalues acquired by the compared algorithms

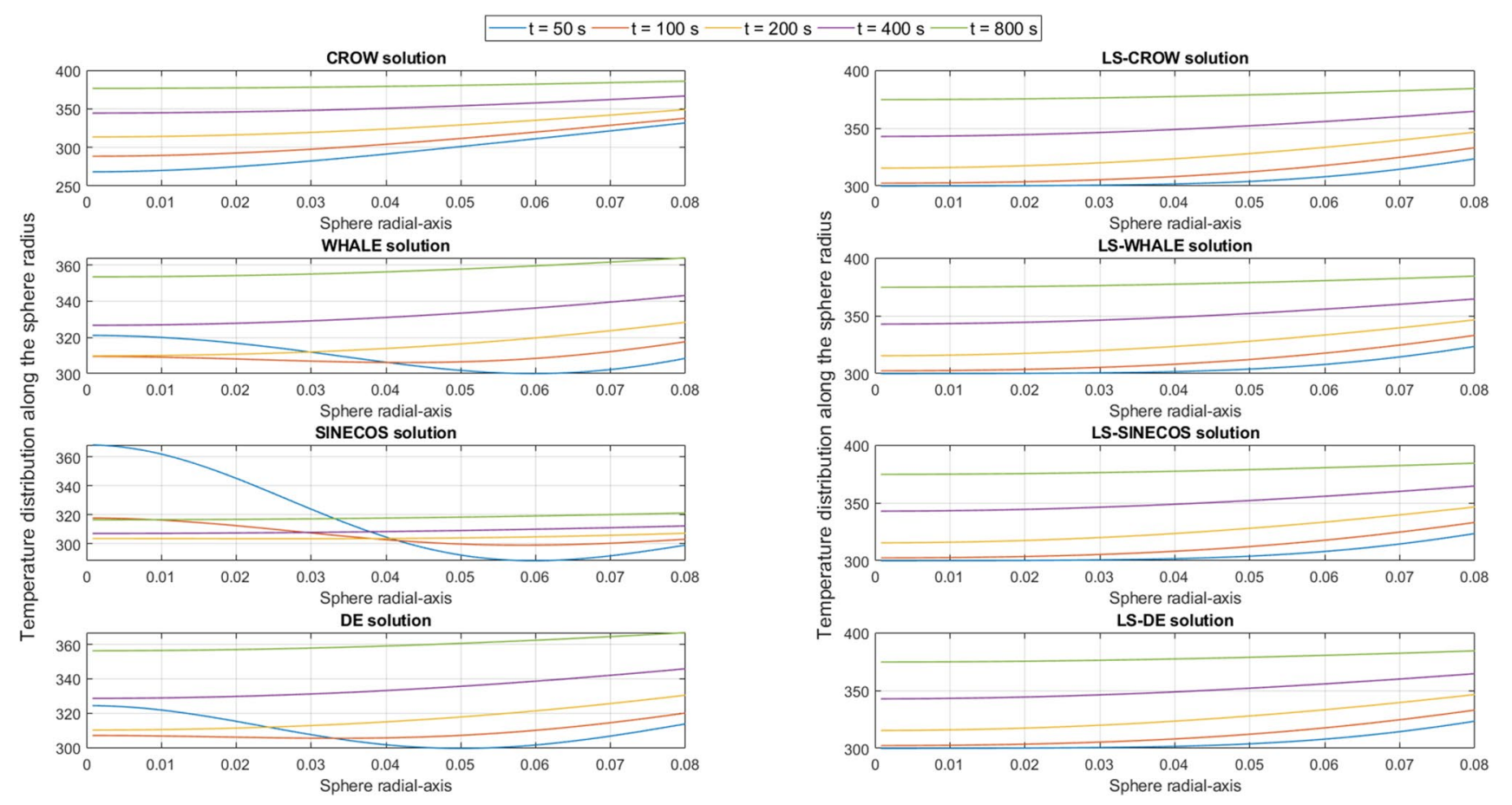

Fig. 18 Temperature distribution along the radius of the sphere heat transfer medium

$\phi^{*}=\sum_{n=1}^{\infty} A_{n} \exp \left(-\beta_{n}^{2} F o\right) \frac{\sin \left(\beta_{n} r^{*}\right)}{\beta_{n} r^{*}}$

where the dimensionless radius is $r^{*}=r / r_{0}$, Fourier number is Fo $=\alpha \cdot t / r_{o^{\prime}}^{2}$ and $A_{n}$ parameter can be expressed by
$A_{n}=\frac{4\left[\sin \left(\beta_{n}\right)-\beta_{n} \cos \left(\beta_{n}\right)\right]}{2 \beta_{n}-\sin \left(2 \beta_{n}\right)}$

where $\beta_{n}$ is the different positive roots of the below defined transcendental equation 


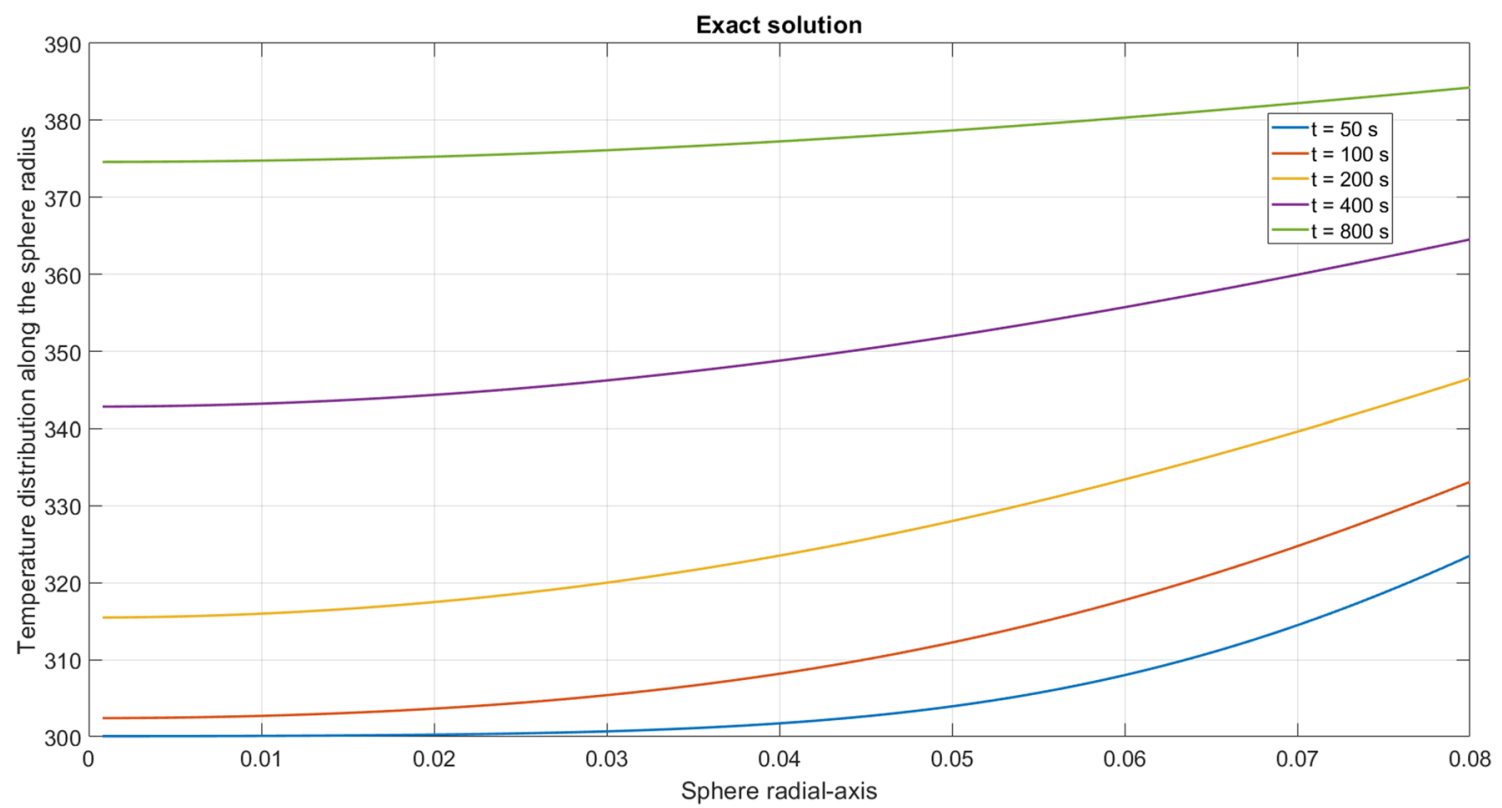

Fig. 19 Time-dependent temperature distribution along the radial axis of a sphere

Table 9 Deviation results in terms of RMSE values to attain the temperature distribution along the sphere radius

\begin{tabular}{lllll}
\hline & Best & SD & Mean & Worst \\
\hline CROW & $1.24 \mathrm{E}+02$ & $3.37 \mathrm{E}+01$ & $1.78 \mathrm{E}+02$ & $2.86 \mathrm{E}+02$ \\
CL-CROW & $4.53 \mathrm{E}-12$ & $4.05 \mathrm{E}-12$ & $6.77 \mathrm{E}-12$ & $2.91 \mathrm{E}-12$ \\
WHALE & $6.23 \mathrm{E}+01$ & $2.34 \mathrm{E}+01$ & $9.65 \mathrm{E}+01$ & $1.49 \mathrm{E}+02$ \\
CL-WHALE & $4.01 \mathrm{E}-09$ & $7.78 \mathrm{E}-07$ & $4.12 \mathrm{E}-07$ & $3.56 \mathrm{E}-06$ \\
SINECOS & $6.22 \mathrm{E}+02$ & $5.41 \mathrm{E}+01$ & $7.66 \mathrm{E}+02$ & $8.84 \mathrm{E}+02$ \\
CL-SINECOS & $9.15 \mathrm{E}-10$ & $4.28 \mathrm{E}-05$ & $7.69 \mathrm{E}-06$ & $2.82 \mathrm{E}-04$ \\
DE & $6.23 \mathrm{E}+01$ & $2.58 \mathrm{E}+01$ & $1.08 \mathrm{E}+02$ & $1.51 \mathrm{E}+02$ \\
CL-DE & $\mathbf{4 . 4 0 E}-12$ & $4.86 \mathrm{E}-12$ & $4.42 \mathrm{E}-12$ & $4.64 \mathrm{E}-12$ \\
\hline
\end{tabular}

Bold values are the best solutions obtained by the corresponding optimization algorithm for the related problem

$1-\beta_{n} \cot \left(\beta_{n}\right)=B i$

where $B i$ is the dimensionless Biot number defined as $B i=h \cdot r_{o} / k$. Equation (28) can be transformed into an unconstrained optimization problem by the following equation in the form of an error function

$f\left(\beta_{n}\right)=1-\beta_{n} \cot \left(\beta_{n}\right)-B i$

The above error function is embedded in Eq. (25) to formulate the objective function of this parameter estimation problem. Assume a sample sphere as a case study whose radius length $\left(r_{0}\right)$ is $0.08 \mathrm{~m}$, thermal conductivity $(k)$ is $15.0 \mathrm{~W} / \mathrm{m} \mathrm{K}$, and thermal diffusivity $(a)$ is $5.0 \mathrm{E}-6 \mathrm{~m}^{2} / \mathrm{s}$ at an initial temperature $\left(T_{i}\right)$ of $300 \mathrm{~K}$, which experiences a thermal interaction between the surrounding ambient $\left(T_{\infty}\right)$ at initially $400 \mathrm{~K}$ having a convective heat transfer coefficient $(h)$ of $200.0 \mathrm{~W} / \mathrm{m}^{2} \mathrm{~K}$. It is aimed to obtain the time-dependent heat conduction along the radial axis of the sample sphere by the compared algorithms for these defined thermal conditions. Figure 17 shows the normalized predicted parameters of eigenvalues obtained by the metaheuristic algorithms. Predictive results found by the local search enhanced methods fully agree well with the exact solutions retained by the Bisection root-finding method for this case. Estimated values are erroneous at the initial steps then deviations rates are decreased as proceeding to the final steps for the remaining metaheuristic algorithms. Figure 18 visualizes the estimated temperature distribution along the sphere radius for each algorithm. It is observed that local search improved methods can capture the time-dependent temperature trend obtained by the Bisection root finding method as shown in Fig. 19. Table 9 reports the statistical deviation results obtained by the compared algorithms for this case. The accuracy of the optimal solutions is much better than those compared to the infinite-wall problem. Local search improved metaheuristics significantly outperform the remaining algorithms in terms of solution accuracy and persistence. Among the improved algorithms, CL-DE slightly surpasses $\mathrm{CL}-\mathrm{CROW}$ in terms of the best fitness value of $4.40 \mathrm{E}-12$ and becomes the best performing improved algorithm for this parameter identification problem. CL-CROW is the second-best method with a minimum objective function value of $4.53 \mathrm{E}-12$, which is followed by the CL-SINECOS method having the best prediction rate of $9.15 \mathrm{E}-10$. 


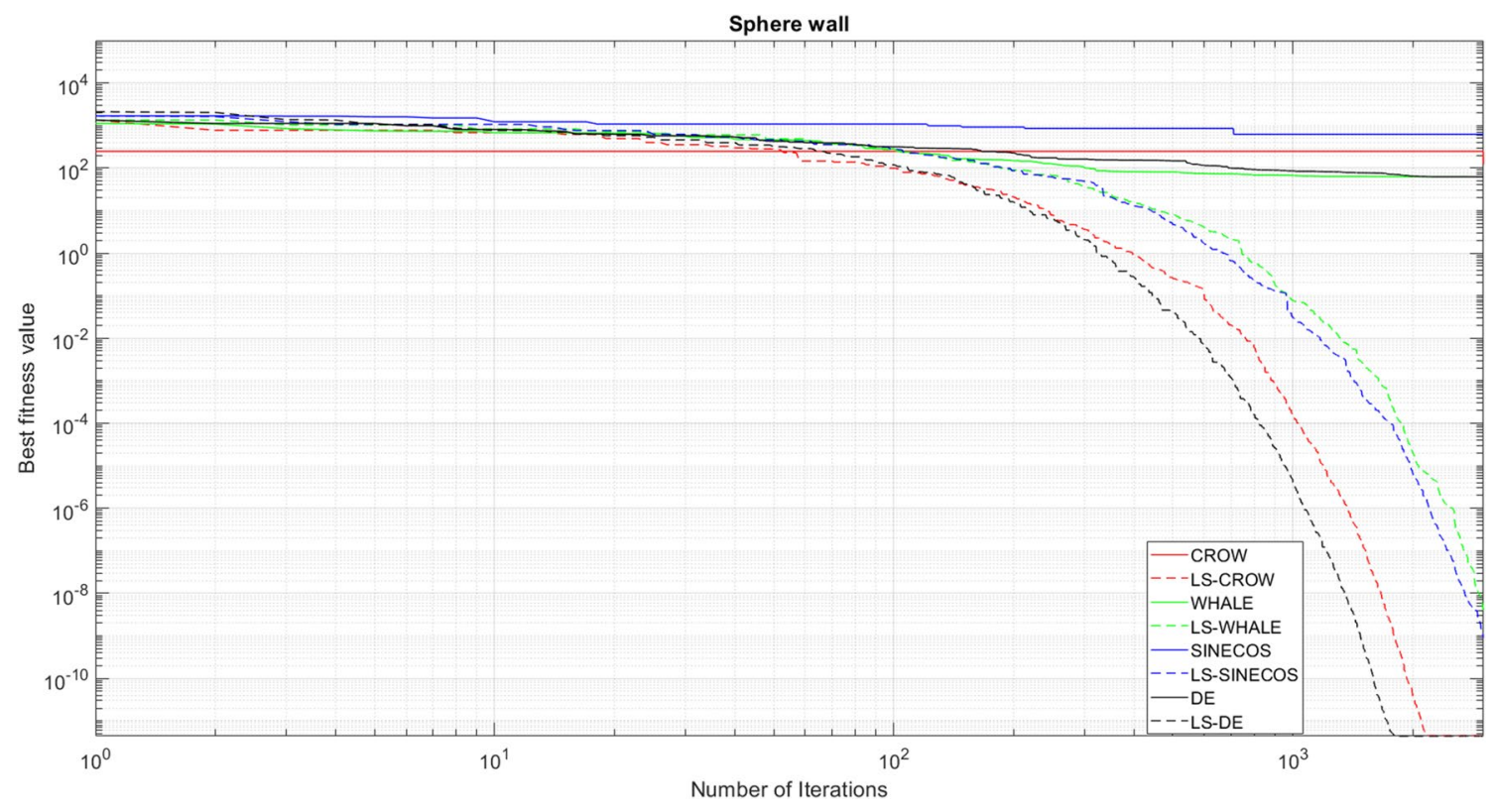

Fig. 20 Evolution histories of the best fitness values of the compared algorithms

CL-WHALE retains the worst estimations between them having the best predictive value of $4.01 \mathrm{E}-09$. Statistical results obtained for the baseline algorithms of CROW, WHAL, SINECOS, and DE are far away from the optimal solution found by local search enhanced optimizers regarding solution quality and robustness. Both $\mathrm{DE}$ and WHALE acquire the best optimum RMSE values of $6.23 \mathrm{E}+01$ while SINECOS yielding the worst predictive results for this case. Convergence curves obtained by the compared metaheuristic algorithms are shown in Fig. 20. After small gradual decreases in the early phases, sharp declines are seen for local search enhanced algorithms at the final stages of the consecutive iterations which are also in line with the inclinations of convergence characteristics observed for the infinite wall problem.

It can be concluded from the comprehensive analysis on different kinds of optimization problems solved in this research study that proposed local search scheme boosted by the hybrid chaotic map allow algorithm for maintaining a plausible and reliable balance between the exploration and exploitation phases, which enables accurate and quick convergence to the optimal solution thanks to the unpredictable nature of the chaotic random numbers. Another favorable characteristic of the proposed local search scheme is its easy and convenient implementation of any type of metaheuristic algorithm. After an exhaustive trial-and-error procedure, while constructing the hybrid chaotic map, it is also interesting to see why some chaotic maps perform well on the specific type of optimization cases while others fail to obtain feasible results. Comprehensive future work should be dedicated to this issue for a better understanding of the true nature of the working mechanisms of the chaotic algorithms. Numerical experiments also indicate that local search methods imposing too much intensification on the promising regions of the search region jeopardize the process of finding an optimal solution and generally get stuck into the local optimum points. Upcoming research studies related to developing local search methods should give a bit more emphasis on the balance between intensification and diversification phases of the algorithm. Furthermore, the developed local search procedure in this study can be applied to the multi-objective real-world design problems to assess its efficiency in complex constrained optimization cases.

\section{Conclusion}

This research study proposes an ensemble local search procedure composed of a hybrid Logistic-Kent chaotic map and a modified version of the Differential Evolution variant. A generic local search procedure is implemented on the famous metaheuristics of Crow Search, Differential Evolution, Whale Optimization, and Sine Cosine optimization algorithms to evaluate the improvement in solution qualities over metaheuristic algorithms with different characteristics. Mentioned algorithms along with their local search enhanced versions have been applied to forty optimization benchmark algorithms involving multimodal and unimodal test problems. Optimal comparative results obtained by the metaheuristic algorithms reveal that amelioration in solution efficiencies is evident and significant 
for the local search enhanced algorithms for most of the cases. It is also seen that can reach the optimal solutions of the benchmark function can be reached within few iterations thanks to the balanced exploration and exploitation capabilities maintained by embedding the local search enhanced method into the pilot metaheuristic algorithms. To assess the prediction accuracy of the proposed local search scheme, two real-world constrained optimization problems with challenging constraints have been solved and corresponding results acquired by the local search improved methods are compared. Remarkable quality improvement in the quantitative results are also observed for these constraint problems by the local search boosted algorithms. Furthermore, the application of the compared metaheuristics algorithms over solving one-dimensional transient heat conduction problems is evaluated. It is comprehended that particularly local search enhanced metaheuristic methods can successfully predict the accurate one-dimensional temperature distribution across the considered heat transfer mediums of infinite wall and sphere and prove their applicability in solving multidimensional nonlinear unconstrained problems. For planned future work, favorable merits of the proposed local search scheme can be utilized on different kinds of metaheuristic algorithms for solving real-world optimization problems with having challenging imposed constraints. Furthermore, utilization of the proposed local search on a metaheuristic optimizer can be useful and practical in application areas such as artificial neural networks and deep learning systems where the global optimum solution should be attained in a fewer number of iterations. Real-time control problems are another branch of the feasible applications of the proposed local search enhanced algorithms where minimum calculation time is highly needed to reduce the computation gap between the current and next time steps which is very crucial and important for stabilizing the projected real-time system.

\section{Compliance with ethical standards}

Conflict of interest The author declares that there is no potential conflict of interest in this work

Open Access This article is licensed under a Creative Commons Attribution 4.0 International License, which permits use, sharing, adaptation, distribution and reproduction in any medium or format, as long as you give appropriate credit to the original author(s) and the source, provide a link to the Creative Commons licence, and indicate if changes were made. The images or other third party material in this article are included in the article's Creative Commons licence, unless indicated otherwise in a credit line to the material. If material is not included in the article's Creative Commons licence and your intended use is not permitted by statutory regulation or exceeds the permitted use, you will need to obtain permission directly from the copyright holder. To view a copy of this licence, visit http://creativecommons .org/licenses/by/4.0/.

\section{References}

1. Kvasov DE, Mukhametzhanov MS (2018) Metaheuristic versus deterministic global optimization algorithms: the univariate case. Appl Math Comput 318:245-259. https://doi.org/10.1016/j. amc.2017.05.014

2. Yousri D, Elaziz MA, Mirjalili S (2020) Fractional-order calculusbased flower pollination algorithm with local search for global optimization and image segmentation. Knowl Based Syst 197:105889. https://doi.org/10.1016/j.knosys.2020.105889

3. Rashedi E, Nezamabadi-pour H, Saryazdi S (2009) GSA: a gravitational search algorithm. Inf Sci 179:2232-2248. https://doi. org/10.1016/j.ins.2009.03.004

4. Kennedy J, Eberhart R (1995) Particle swarm optimization. In: Proceedings of ICNN'95-international conference on neural networks, vol 4, pp 1942-1948

5. Zhang Z, Ding S, Jia W (2019) A hybrid optimization algorithm based on cuckoo search and differential evolution for solving constrained engineering problems. Eng Appl Artif Intell 85:254268. https://doi.org/10.1016/j.engappai.2019.06.017

6. Singh N, Son LH, Chiclana F, Magnot J-P (2020) A new fusion of salp swarm with sine cosine for optimization of non-linear functions. Eng Comput 36:185-212. https://doi.org/10.1007/s0036 6-018-00696-8

7. Tavazoei MS, Haeri M (2007) Comparison of different onedimensional maps as chaotic search pattern in chaos optimization algorithms. Appl Math Comput 187:1076-1085. https://doi. org/10.1016/j.amc.2006.09.087

8. Alatas B, Akin E, Ozer AB (2009) Chaos embedded particle swarm optimization algorithms. Chaos Soliton Fract 40:1715-1734. https://doi.org/10.1016/j.chaos.2007.09.063

9. Gandomi AH, Yang X-S, Talatahari S, Alavi AH (2013) Firefly algorithm with chaos. Commun Nonlinear Sci 18:89-98. https://doi. org/10.1016/j.cnsns.2012.06.009

10. Wang G-G, Guo L, Gandomi AH et al (2014) Chaotic Krill Herd algorithm. Inf Sci 274:17-34. https://doi.org/10.1016/j. ins.2014.02.123

11. Mirjalili S, Gandomi AH (2017) Chaotic gravitational constants for the gravitational search algorithm. Appl Soft Comput 53:407-419. https://doi.org/10.1016/j.asoc.2017.01.008

12. Rezaee Jordehi A (2015) A chaotic artificial immune system optimisation algorithm for solving global continuous optimisation problems. Neural Comput Appl 26:827-833. https://doi. org/10.1007/s00521-014-1751-5

13. Sayed Gl, Tharwat A, Hassanien AE (2019) Chaotic dragonfly algorithm: an improved metaheuristic algorithm for feature selection. Appl Intell 49:188-205. https://doi.org/10.1007/s1048 9-018-1261-8

14. Moradi M, Parsa S (2019) An evolutionary method for community detection using a novel local search strategy. Phys $A$ 523:457-475. https://doi.org/10.1016/j.physa.2019.01.133

15. Qu BY, Liang JJ, Suganthan PN (2012) Niching particle swarm optimization with local search for multi-modal optimization. Inf Sci 197:131-143. https://doi.org/10.1016/j.ins.2012.02.011

16. Toksari MD (2016) A hybrid algorithm of ant colony optimization (ACO) and iterated local search (ILS) for estimating electricity domestic consumption: case of Turkey. Int J Electric Power 78:776-782. https://doi.org/10.1016/j.ijepes.2015.12.032

17. Xia X, Liu J, Hu Z (2014) An improved particle swarm optimizer based on tabu detecting and local learning strategy in a 
shrunk search space. Appl Soft Comput 23:76-90. https://doi. org/10.1016/j.asoc.2014.06.012

18. Yildiz YE, Topal AO (2019) Large scale continuous global optimization based on micro differential evolution with local directional search. Inf Sci 477:533-544. https://doi.org/10.1016/j. ins.2018.10.046

19. Gao K, Zhang Y, Sadollah A, Su R (2016) Optimizing urban traffic light scheduling problem using harmony search with ensemble of local search. Appl Soft Comput 48:359-372. https://doi. org/10.1016/j.asoc.2016.07.029

20. Storn R, Price K (1997) Differential evolution-a simple and efficient heuristic for global optimization over continuous spaces. J Global Optim 11:341-359. https://doi.org/10.1023/A:10082 02821328

21. Askarzadeh A (2016) A novel metaheuristic method for solving constrained engineering optimization problems: crow search algorithm. Comput Struct 169:1-12. https://doi.org/10.1016/j. compstruc.2016.03.001

22. Mirjalili S (2016) SCA: a sine cosine algorithm for solving optimization problems. Knowl Based Syst 96:120-133. https://doi. org/10.1016/j.knosys.2015.12.022

23. Mirjalili S, Lewis A (2016) The whale optimization algorithm. Adv Eng Softw 95:51-67. https://doi.org/10.1016/j.advengsoft .2016.01.008

24. Luo Y, Yu J, Lai W, Liu L (2019) A novel chaotic image encryption algorithm based on improved baker map and logistic map. Multimed Tools Appl 78:22023-22043. https://doi.org/10.1007/ s11042-019-7453-3
25. Demir FB, Tuncer T, Kocamaz AF (2019) Lojistik-Gauss Harita Tabanlı Yeni Bir Kaotik Sürü Optimizasyon Yöntemi. Bilgisayar Bilimleri 4:47-53

26. Pan S, Wei J, Hu S (2020) A novel image encryption algorithm based on hybrid chaotic mapping and intelligent learning in financial security system. Multimed Tools Appl 79:9163-9176. https://doi.org/10.1007/s11042-018-7144-5

27. Demir FB, Tuncer T, Kocamaz AF (2020) A chaotic optimization method based on logistic-sine map for numerical function optimization. Neural Comput Appl. https://doi.org/10.1007/s0052 1-020-04815-9

28. Mlakar U, Brest J, Fister I (2016) A study of chaotic maps in differential evolution applied to gray-level image thresholding. In: 2016 IEEE symposium series on computational intelligence (SSCl), pp 1-8

29. van den Bergh F, Engelbrecht AP (2006) A study of particle swarm optimization particle trajectories. Inf Sci 176:937-971. https://doi.org/10.1016/j.ins.2005.02.003

30. Pant $M$, Thangaraj R, Singh VP (2009) Optimization of mechanical design problems using improved differential evolution algorithm. IJRTER 1:5

31. Andrei N (2013) Nonlinear optimization applications using the GAMS technology. Springer, Boston

32. Schneider PJ (1957) Conduction heat transfer. SessaBks (ABAA, ILAB). Accessed 16 Jun 2020

Publisher's Note Springer Nature remains neutral with regard to jurisdictional claims in published maps and institutional affiliations. 